\title{
Polymer Injectivity: Investigation of Mechanical Degradation of Enhanced Oil Recovery Polymers Using In-Situ Rheology
}

\author{
Badar Al-Shakry $1,2, *\left(\mathbb{0}\right.$, Tormod Skauge ${ }^{3} \mathbb{C}^{\text {, Behruz Shaker Shiran }}{ }^{2}$ and Arne Skauge ${ }^{1,2,3}$ \\ 1 Department of Chemistry, University of Bergen, Allegaten 41, 5007 Bergen, Norway; Arne.Skauge@uib.no \\ 2 NORCE Energy, CIPR, Nygårdsgaten 112, 5008 Bergen, Norway; besh@norceresearch.no \\ 3 Energy Research Norway, Allegaten 41, 5007 Bergen, Norway; Tormod.Skauge@energyresearch.no \\ * Correspondence: Badar.Al-Shakry@uib.no; Tel.: +47-5558-3672
}

Received: 22 November 2018; Accepted: 19 December 2018; Published: 24 December 2018

\begin{abstract}
Water soluble polymers have attracted increasing interest in enhanced oil recovery (EOR) processes, especially polymer flooding. Despite the fact that the flow of polymer in porous medium has been a research subject for many decades with numerous publications, there are still some research areas that need progress. The prediction of polymer injectivity remains elusive. Polymers with similar shear viscosity might have different in-situ rheological behaviors and may be exposed to different degrees of mechanical degradation. Hence, determining polymer in-situ rheological behavior is of great significance for defining its utility. In this study, an investigation of rheological properties and mechanical degradation of different partially hydrolyzed polyacrylamide (HPAM) polymers was performed using Bentheimer sandstone outcrop cores. The results show that HPAM in-situ rheology is different from bulk rheology measured by a rheometer. Specifically, shear thickening behavior occurs at high rates, and near-Newtonian behavior is measured at low rates in porous media. This deviates strongly from the rheometer measurements. Polymer molecular weight and concentration influence its viscoelasticity and subsequently its flow characteristics in porous media. Exposure to mechanical degradation by flow at high rate through porous media leads to significant reduction in shear thickening and thereby improved injectivity. More importantly, the degraded polymer maintained in-situ viscosity at low flow rates indicating that improved injectivity can be achieved without compromising viscosity at reservoir flow rates. This is explained by a reduction in viscoelasticity. Mechanical degradation also leads to reduced residual resistance factor (RRF), especially for high polymer concentrations. For some of the polymer injections, successive degradation (increased degradation with transport length in porous media) was observed. The results presented here may be used to optimize polymer injectivity.
\end{abstract}

Keywords: enhanced oil recovery (EOR); polymer flooding; injectivity; rheology; viscoelasticity; non-Newtonian flow; mechanical degradation; HPAM

\section{Introduction}

In today's oil industry, chemical enhanced oil recovery techniques such as polymer flooding play a substantial role in promoting oil production. This is attributed to the achieved improvement on sweep efficiency that boosts oil production over conventional waterflooding. In such a process, water-soluble polymers are added to viscosify injected water in order to achieve lower viscosity contrast between injected water and displaced oil, and therefore a favorable mobility ratio [1]. Besides mobility control, high viscosity polymers are required for better conformance control relevant to heterogeneous reservoirs with high permeability variations such as the presence of thief zones [2]. 
There are two types of polymers suit enhanced oil recovery (EOR) applications which are: biopolymers, e.g., xanthan, and synthetic polymers, e.g., partially hydrolyzed polyacrylamide (HPAM). Regardless of the nature and differences in the molecular structure of these two polymers, polymer viscosity is the main physical property in the context of polymer flooding. Polymer viscosity depends on polymer molecular structure, molecular weight [3], polymer concentration [4], salinity $[5,6]$, temperature $[7,8]$, degree of hydrolysis [9], $\mathrm{pH}$ [10], flow model and type of forces dominating the flow [11].

While xanthan is well-known to be viscous dominated, HPAM is strongly influenced by both viscous and elastic properties [12]. It is essential to understand the significance and consequences when HPAM fluids become elastic dominated. HPAM viscoelasticity is important for many applications in the oil industry in general (e.g., drag reduction, drilling, etc.) and specifically in EOR applications such as polymer flooding [1,13-16], Low Salinity Polymer (LSP) flooding [17,18] and Alkaline-Surfactant-Polymer (ASP) flooding $[19,20]$. In polymer flooding, HPAM viscoelasticity is believed to contribute to higher oil recovery in general and some claim that it might reduce residual oil saturation due to promoting pulling effect mechanisms [21-24]. HPAM shear thickening behavior may, in some cases, contribute to improving front stability and oil recovery [25]. On the other hand, the significant pressure gradient associated with shear thickening phenomena can limit polymer injection, cause wellbore damage or fracturing. The influence of mechanical degradation on shear rheology will be discussed in this paper.

\section{Polymer Injectivity and Mechanical Degradation}

Polymer injectivity is a measure of how easily a polymer solution can be delivered into a reservoir formation [26]. It is also a measure of how fast polymer solution can be injected and propagate through the reservoir. It is a critical task because a decline in injectivity can turn the predicted cashflow of polymer flooding projects negatively $[27,28]$. This is basically due to the delay of oil production or high pumping cost. Both aforementioned polymers (xanthan and HPAM) may suffer from injectivity problems for different reasons. For instance, the presence of microgels and impurities in xanthan may limit its injectivity [29]. However, HPAM viscoelasticity and retention are the main factors that restrict its injectivity. The design of polymer flooding projects has to cover some key aspects such as reservoir formation, oil saturation, injection strategy, polymer rheology, degradation, compatibility with other chemicals, economy, etc. [30]. This paper intends to investigate some of these aspects such as the link between polymer rheology and degradation.

The theories and observations associated with the characterization of flow of biopolymers such as xanthan are typically united in that xanthan has pseudoplastic rheological behavior in porous media similar to that predicted in pure shear flow such in the rheometer [31-35]. However, the situation is more complicated for HPAM due to its viscoelastic nature and the complexity of porous media. Despite the rich literature of polymer flow in porous media, the theoretical interpretations are still conflicting on the analysis of the observed HPAM in-situ flow behaviors. HPAM polymers are well-known to have high polydispersity index [36] and possess long relaxation time. HPAM has a flexible molecular structure and highly sensitive to shear environments. When HPAM flows in porous media, it is exposed to both shear and elongational deformations as it is transported through converging-diverging $(C \leftrightarrow D)$ flow channels [36,37]. This results in successive expansion and contraction $(\mathrm{E} \leftrightarrow \mathrm{C})$ of polymer conformation as it flows through porous media. Figure 1 illustrates a schematic representation of typical flow regions that are exhibited by HPAM with respect to shear rate. The polymer exhibits near-Newtonian behavior at which its apparent viscosity is independent of imposed shear rates $\left(\dot{\gamma}<\dot{\gamma}_{\mathrm{c} 1}\right)$. As the shear rate increases further, apparent viscosity decreases and the polymer solution exhibits shear thinning behavior. During shear thinning, polymer molecules start to disentangle with increasing shear rate until approaching another Newtonian plateau at which the state of disentanglement is very high. However, above $\dot{\gamma}_{\mathrm{c} 2}$, the extensional flow becomes predominant at which polymer chains have insufficient time to recoil and align with the flow causing coil-stretch $(C \leftrightarrow S)$ transition that yields in a gradual increase of apparent viscosity with shear rate. The normal 
stresses that are responsible for chain stretch cause a rise in the extensional viscosity and consequently cause pressure buildup and high apparent viscosity (shear thickening behavior). If the stretch rates that are associated with shear thickening behavior are high enough, chain stretch might evolve into chain fragmentation. Chain scissions due to mechanical degradation yields in viscosity loss as can be seen at high shear rates displayed in Figure 1. These flow phenomena are detailed elsewhere [11,37-40]. The large strain forces cause large molecules to shear preferentially. Literature reviews on polymer mechanical degradation [41,42] showed that the assessment of mechanical degradation is complex, particularly in the presence of entanglements and concentrated conformational regimes. It is very important to understand how HPAM macromolecules contribute to changing its flow phenomena and increasing its apparent viscosity at high flow rates that scales several folds higher than predicted in simple shear flow such that generated by the rheometer.



Figure 1. Schematic diagram of HPAM apparent viscosity vs shear rate.

In addition to HPAM shear stability discussed above, the dimensions and conformation of HPAM molecules strongly depend on the salt concentration and types of TDS existing in solution. HPAM is a negatively charged polymer, and therefore at the presence of salts, the repulsion forces among polymer chains decrease due to shielding negative charges which cause coiling-up phenomena [43]. In some cases, the presence of salts in high concentration might lead to phase separation (e.g., gel-formation) [44]. The reduction in viscosity due to salinity is more pronounced at the presence of divalent cations (e.g., $\mathrm{Ca}^{2+}, \mathrm{Mg}^{2+}$, etc.) compared to that of monovalent cations like $\mathrm{Na}^{+}[43]$.

The previous study by Skauge et al. [45] demonstrated a combination of experiments for investigating the contributions of polymer molecular weight and concentration conformational regimes to its rheological properties. The measurements included shear viscosity (rotational rheometer), dynamic viscosity (small amplitude oscillatory shear SAOS) and in-situ rheology (Bentheimer cores). The study classified the investigated polymer solutions into different conformational regimes; dilute, semi-dilute, concentrated semi-dilute and gel solutions, based on critical overlap concentration $\left(C^{*}\right)$. SAOS measurements indicated whether the polymer solution is viscous or elastic dominated at a particular concentration. The initial studies showed a correlation between bulk elastic modulus $G^{\prime}$ and apparent shear thickening. The more elastic polymer exhibited higher resistance factor in porous media. The study also revealed that polymer conformation regime has a high influence on its in-situ rheological behavior. Shear viscosity data showed that mechanical degradation was high for high Mw polymer dissolved in high salinity brine. Also, mechanical degradation was lower for concentrated 
solutions. Recent review with current knowledge on HPAM polymers flow in porous media concerning theoretical and experimental aspects is given by Skauge et al. [46].

One of the most critical aspects of HPAM polymer is mechanical degradation. Such an effect directly influences polymer viscosifying efficiency as well as alters its rheological properties. Both HPAM shear thickening behavior and mechanical degradation are well reported [12,47-50]. Mechanical degradation might occur along with the onset of shear thickening [51]. Onset of shear thickening has received a great attention in the literature as it is an indication of viscoelasticity in porous medium [50,52-55]. Any alteration of the molecular structure of HPAM through exposing it to shear rate above or below the onset of shear thickening may change its apparent shear thickening behavior [56]. Preshearing polymer by exposing HPAM to wellbore mechanical degradation is a suggested approach to improve its viscoelastic properties which promotes its injectivity [57]. Despite the efforts that have been made to understand and model polymer mechanical degradation, the dependence of polymer mechanical degradation on polymer Mw, MWD, concentration, and polymer transport distance in porous media requires more investigation [12,26,49].

The impact of mechanical degradation on polymer average $\mathrm{Mw}$ and molecular weight distribution (MWD) were examined in different studies. For example, Seright et al. [58] investigated the mechanical degradation effect on polymer Mw and MWD by using gel permeation liquid chromatography (GPC). The degraded solutions showed narrower MWD compared to that of undegraded samples. Hence, degraded polymer solution has a lower polydispersity. This concept was also confirmed by size exclusion chromatography (SEC) [59] and asymmetric flow field-flow fractionation (AF4) [30,60-62]. Reduction of Mw or change in MWD is the reason for the observed reduction in screen factor $[12,63]$ that correlates with reduction of resistance factor as well.

Noïk et al. [36] investigated the effect of Mw, concentration and different types of solvents on the mechanical degradation of HPAM in short glass cylinders packed with sand particles. The high Mw polymers were subjected to wellbore mechanical degradation through successive reinjection into porous media. Reinjection process represents the evolution of degradation as a function of residence time or the length of porous media. Degradation was assessed by observing the change in intrinsic viscosity of solutions before and after degradation. They found that the degree of degradation is independent of concentration for dilute solutions and was only dependent on Mw. However, for concentrated solutions, the degradation increases with concentration and has less dependency on average Mw.

Several studies attributed HPAM mechanical degradation to the polymer degradation in sandface, and therefore, understated the effect of polymer residence time or transported distance in porous media $[12,38,49,64,65]$. For example, Maerker [12] attributed mechanical degradation to the first 0.5 inch of porous media while Warner [64] attributed it to the first inch of unperforated wellbore based on studies performed in Berea rock. Müller et al. [51] reported that the mechanical degradation of HPAM polymers increased with travel distance and progressively degraded until reached an asymptotic value that depends on the stretch rate which related to Reynold's number. A recent study given by Jouenne et al. [66] highlighted the observation of mechanical degradation at entry face and limited it for the first $6 \mathrm{~mm}$ of porous media based on studies performed in a ceramic disk. However, Al-Shakry et al. [67] conducted experimental studies using HPAM polymers that showed high Mw polymer underwent successive degradation as reinjected into the porous media. This suggests that the degree of degradation may depend on exposure time and number of exposures to high strain beside polymer Mw and concentration. The findings were also in line with Åsen et al. [68]. The dependence of mechanical degradation on travelled distance in porous media has a significant practical consequences specifically when considering the effect on large scale medium such as field conditions. These observations were also supported by other studies based on analyzing shear viscosity data alone [69-71].

Despite the current efforts made both experimentally and theoretically to clarify the problem of mechanical degradation, the current understanding is not complete, and further analyses are required. 
This paper extends our previous work [67] that provides a basis for this study on experimental investigation of the impact of mechanical degradation on polymer in-situ rheology. This work extends the analyses to address the influence of polymer physicochemical properties, particularly molecular weight and concentration on polymer mechanical degradation and its in-situ behavior. Particular attention was given for the impact of preconditioning the polymer solution prior to injection into the porous media on polymer in-situ rheology. The study was performed in a realistic porous medium using high preamble linear Bentheimer core plugs. The results from this paper give an insight into in-situ rheological behavior of commercial HPAM polymers, which may be beneficial in polymer screening and designing of polymer flooding EOR operations. The results from this study may also serve as useful input for simulation models.

\section{Material and Methods}

\subsection{Synthetic Brine}

Synthetic brine of $1 \mathrm{wt} . \% \mathrm{NaCl}$ was prepared and filtered through a $0.45 \mu \mathrm{m}$ cellulose nitrate filter. The brine composite ( $\mathrm{NaCl}$ powder) was obtained from Sigma-Aldrich (Munich, Germany). The prepared filtered brine was employed in the preparation of bulk polymer solutions, core saturation and permeability measurements.

\subsection{Polymer Preparation}

Three types of partially hydrolyzed polyacrylamide (HPAM) with 30\% degree of hydrolysis were employed in this study with different concentrations as shown in Table 1 . These polymers are Flopaam 3330 s, $3430 \mathrm{~s}$ and $3630 \mathrm{~s}$ which are donated as polymer A, B and C, respectively. These polymers were received in powder form from SNF Floerger. Each polymer was prepared with low and high concentration within semi-dilute region to provide a low and high degree of entanglements, respectively. The selected concentration for each polymer was based on overlap concentration $\left(C^{*}\right)$ determined in earlier studies performed by Skauge et al. [45].

The polymer stock solution of $5000 \mathrm{ppm}$ was prepared by gradually dissolving $3.0 \mathrm{~g}$ of polymer powder into the vortex of $1 \mathrm{wt} . \% \mathrm{NaCl}$ brine under vigorous stirring until the vortex became invisible. The polymer solution was left under slow mixing at a stirring speed of $150 \mathrm{rpm}$ for at least $24 \mathrm{~h}$ before dilution into required concentration. The polymer was thoroughly sealed during the preparation. The prepared aqueous polymer solution was incubated at $5{ }^{\circ} \mathrm{C}$ inside a fridge and used within two weeks of preparation to avoid any chance of chemical degradation.

Table 1. Molecular weights and concentrations of polymers.

\begin{tabular}{|c|c|c|c|}
\hline Polymer & Polymer (Flopaam) Type & Molecular Weight $\left(10^{6} \mathrm{~g} / \mathrm{mol}=\mathrm{MDa}\right)$ & $\begin{array}{l}\text { Polymer Concentration } \\
(\mathrm{mg} / \mathrm{L}=\mathrm{ppm})\end{array}$ \\
\hline A & $3330 \mathrm{~s}$ & 8 & $\begin{array}{l}1000 \\
4000\end{array}$ \\
\hline B & $3430 \mathrm{~s}$ & 12 & $\begin{array}{l}1000 \\
3000\end{array}$ \\
\hline $\mathrm{C}$ & $3630 \mathrm{~s}$ & 18 & $\begin{array}{c}500 \\
1000\end{array}$ \\
\hline
\end{tabular}

\subsection{Shear Viscosity Measurements}

Shear viscosity measurements were carried out at room temperature $\left(22^{\circ} \mathrm{C}\right)$ by using a Kinexus Pro Rheometer (Malvern, UK). The rheometer is equipped with different geometries which make the measurements more accurate and convenient to conduct based on fluid types and viscosity. Hence, double-gap geometry was used during the measurements of viscosities lower than $10 \mathrm{cP}$ and cone-plate 
geometry was used for measuring viscosities higher than $10 \mathrm{cP}$. The viscosity measurements were fitted by power law model given below:

$$
\eta=\mathrm{K} \dot{\gamma}^{\mathrm{n}-1}
$$

where, $\eta$ is shear viscosity $(c P), \dot{\gamma}$ is shear rate $\left(s^{-1}\right), K$ is the consistency index $(c P . s(n-1))$ and $n$ is the flow behavior index (dimensionless).

\subsection{Porous Medium}

The experiments were conducted in linear Bentheimer outcrop cores with an average length and diameter of $10 \mathrm{~cm}$ and $3.8 \mathrm{~cm}$, respectively. Similar cores of Bentheimer with shorter length of $5 \mathrm{~cm}$ were also used for prefiltering and preshearing processes. Details of each core are given in results and discussion section.

\subsection{Experimental Procedures}

The experimental setup displayed in Figure 2 mainly consists of Quizix-QX dual piston pump, transfer cylinder, core holder, pressure transducers, back pressure regulator and effluent collector. Note that, backpressure regulator was used during permeability measurements to dissolve any air in the setup and it was removed during polymer injection to avoid polymer degradation.

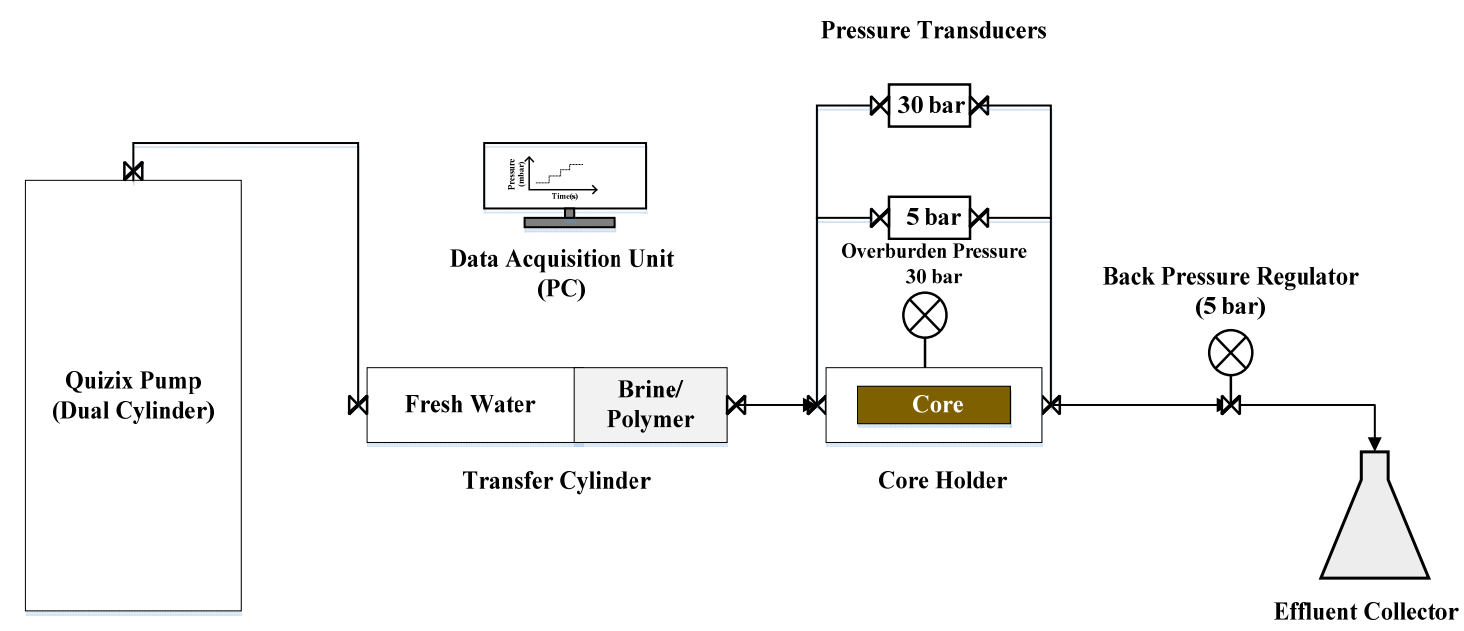

Figure 2. Schematic diagram of core flooding apparatus used for in-situ rheology experiments.

Core flood experiments were conducted at room temperature $\left(22^{\circ} \mathrm{C}\right)$ and consisted of three stages as detailed in the previous study [67]. The core flood procedure was performed as follows:

\subsubsection{Brine Pre-Flush}

Before injecting the brine, the core plugs were vacuumed and saturated with brine for at least two days to ensure achieving ionic equilibrium between the core plug and brine followed by porosity measurements. Then, the core plug was mounted in the core holder and brine was injected at various flow rates to measure absolute permeability $\left(\mathrm{K}_{\mathrm{abs}}\right)$ which was calculated according to Darcy's law (Equation (2)):

$$
\mathrm{K}_{\mathrm{abs}}=\frac{\mathrm{Q} \times \eta \times \mathrm{L}}{\Delta \mathrm{P} \times \mathrm{A}}
$$

where, $Q$ is injection flow rate, $\eta$ is fluid viscosity, $\Delta P$ is pressure drop across the core, $L$ and $A$ are core length and cross-sectional area, respectively. By considering Darcy velocity $\left(\mathrm{v}_{\mathrm{D}}\right)$ which is also 
known as superficial flow velocity as Q/A, the average or interstitial velocity (v) is given in Equation (3), where $\phi$ is the porosity of porous media:

$$
v=\frac{v_{D}}{\phi}
$$

Darcy velocity $\mathrm{v}_{\mathrm{D}}$ was also applied to calculate reservoir shear rate $\dot{\gamma}$. A conventional formula was used to estimate reservoir formation shear rate [65]:

$$
\dot{\gamma}=\alpha \frac{4 v_{\mathrm{D}}}{\sqrt{8 \mathrm{~K}_{\mathrm{abs}} \phi}}
$$

where, $\alpha$ is formation shape factor which is assumed 2.5 for Bentheimer sandstone $[1,38]$.

\subsubsection{Polymer Injection}

The investigated polymers were pretreated first before injection into the main cores. Pretreatment processes consisted of prefiltering, reinjecting and preshearing as illustrated in Figure 3. Pre-filtering and preshearing processes were performed on short cores $(\mathrm{L}=5 \mathrm{~cm})$ at low and high flow rates, respectively. Reinjected polymer has been prefiltered first in short core then sheared at high flow rate in long core $(\mathrm{L}=10 \mathrm{~cm})$. The flow rate used in prefiltering process was $\left(\mathrm{Q}=0.5 \mathrm{cc} / \mathrm{min}, \mathrm{v}_{\mathrm{D}}=0.6 \mathrm{~m} / \mathrm{day}\right)$ whereas the flow rates applied in preshearing and reinjecting are given in Table 2. Recall that the purpose of prefiltering was to remove any microgel in the solution and filter out any possible large Mw species. This step represents available commercial polymers that are utilized in field applications. Prefiltered polymer solutions also serve as a baseline for comparison with presheared and reinjected solutions. Preshearing was carried out to induce partial degradation in which large Mw species in the solution are likely filtered and mechanically degraded to lower Mw species. While reinjection was designed to evaluate the evolution of polymer degradation with respect to the residence time of polymer solution, core characteristic length, number of passes and multi entry effects.

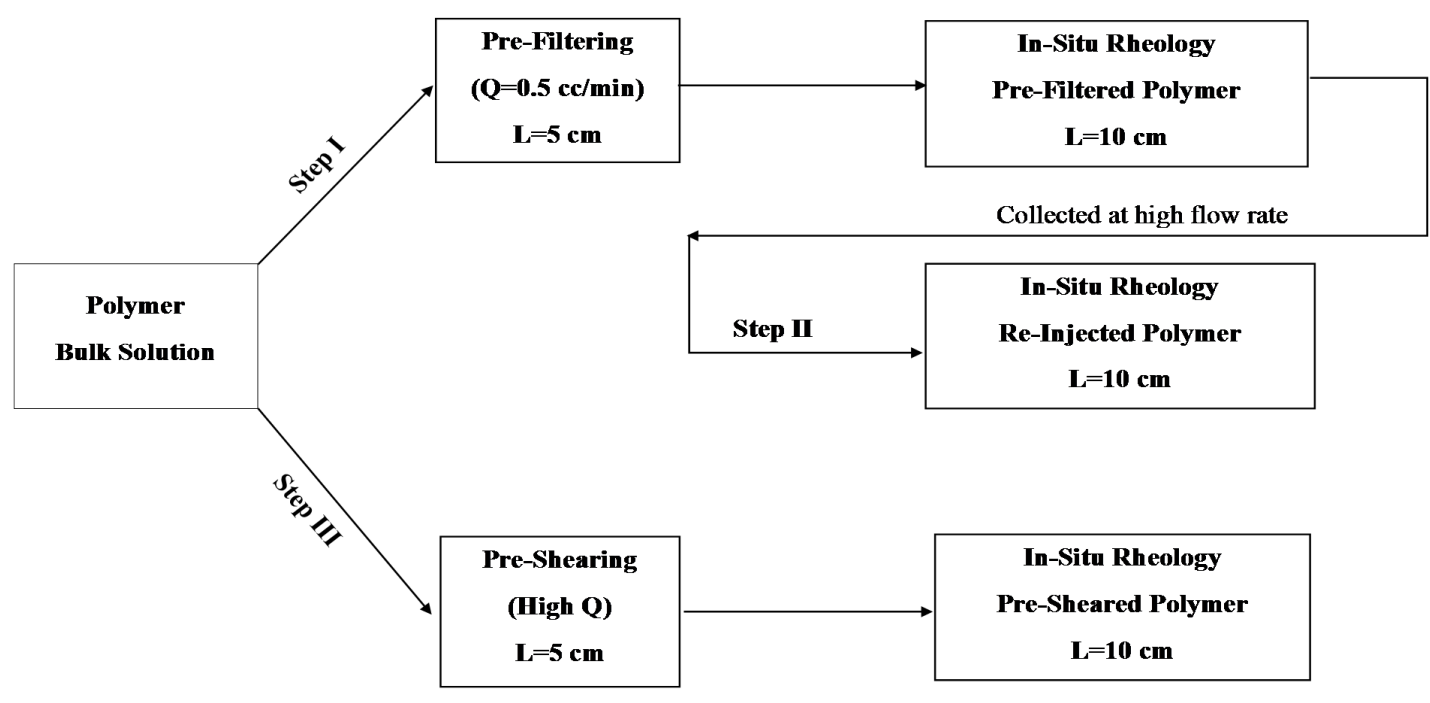

Figure 3. Polymer pretreatment processes.

Table 2. Applied flow rates for presheared and reinjected polymer solutions.

\begin{tabular}{ccccccc}
\hline Polymer & \multicolumn{2}{c}{ A } & \multicolumn{2}{c}{ B } & \multicolumn{2}{c}{ C } \\
\hline Concentration (ppm) & 1000 & 4000 & 1000 & 3000 & 500 & 1000 \\
$\mathrm{Q}(\mathrm{cc} / \mathrm{min})$ & 26 & 22 & 15 & 7 & 12 & 6 \\
$v_{D}(\mathrm{~m} /$ day $)$ & 33.5 & 28.4 & 19.4 & 9.0 & 15.5 & 7.7 \\
\hline
\end{tabular}


Pretreated polymer solution was injected into the main core at low flow rate $(\mathrm{Q}=0.5 \mathrm{cc} / \mathrm{min}$, $\mathrm{v}_{\mathrm{D}}=0.6 \mathrm{~m} /$ day) for at least $2 \mathrm{PV}$. This was performed to satisfy polymer adsorption level in the core and achieving steady-state condition (stable differential pressure over time). Then, the injection flow rate was increased gradually to achieve highest flow rate given in Table 2 and then decreased in a stepwise manner from highest to lowest flow rate. The polymer injection over wide flow rates was performed to simulate the velocities that are anticipated near wellbore region and deep in the reservoir. Resistance factor (RF) was calculated as follows [72]:

$$
\mathrm{RF}=\frac{\Delta \mathrm{P}_{\mathrm{p}}}{\Delta \mathrm{P}_{\mathrm{w}}}
$$

where, $\Delta \mathrm{P}_{\mathrm{p}}$ is the pressure drop of the polymer during polymer flow and $\Delta \mathrm{P}_{\mathrm{w}}$ is the pressure drop of the brine before polymer flow in the porous medium.

After injecting for at least 1 PV for each step rate and steady-state condition was achieved, effluent samples of were collected at different flow rates and their shear viscosity was measured by the rheometer. The following equation was used to express the change in shear viscosity (mechanical degradation) [66]:

$$
\operatorname{Deg}(\%)=\frac{\eta_{i}-\eta_{e}}{\eta_{i}-\eta_{w}} \times 100
$$

where, $\eta_{i}$ is injected solution viscosity, $\eta_{\mathrm{e}}$ is effluent viscosity and $\eta_{\mathrm{w}}$ is brine viscosity which was measured to be $1.04 \mathrm{cP}$. The viscosity data used in this equation were measured at the shear rate of $10 \mathrm{~s}^{-1}$.

\subsubsection{Brine Post-Flush}

After terminating the polymer injection, tapering was performed by injecting $5 \mathrm{PV}$ of diluted polymer effluent with 50 and $25 \%$ of initial effluent concentration. During tapering the injection of the diluted polymer was performed at low flow rate $\left(\mathrm{Q}=1.0 \mathrm{cc} / \mathrm{min}, \mathrm{v}_{\mathrm{D}}=1.3 \mathrm{~m} /\right.$ day) for $1 \mathrm{PV}$ then gradually increased to higher flow rates. After tapering with polymer, brine was injected at low flow rate $\left(\mathrm{Q}=0.5 \mathrm{cc} / \mathrm{min}, \mathrm{v}_{\mathrm{D}}=0.6 \mathrm{~m} /\right.$ day $)$ for $1 \mathrm{PV}$ then the injection rate was increased in a stepwise manner. The final permeability to brine was measured after flushing $5 \mathrm{PV}$ of brine at high rates proceeded by two steps of tapering. Tapering was performed in an effort to approach 'true' residual resistance factor (RRF) which was calculated using Equation (7) [72]:

$$
R R F=\frac{K_{w i}}{K_{w f}}
$$

where, $\mathrm{K}_{\mathrm{wi}}$ and $\mathrm{K}_{\mathrm{wf}}$ are the absolute permeability's to brine before and after polymer flow in porous media, respectively. These values were calculated by using Equation (2).

\section{Results and Discussion}

\subsection{Shear Viscosity}

The bulk shear viscosity of polymer solutions was measured in the rheometer at a wide range of shear rates as shown in Figure 4. At the mid-range of shear rates, all the solutions exhibited a predominant shear thinning behavior. The measurements were showed a good fit to the power law model (Equation (1)) using the fitting parameters given in Table 3. Concentrated solutions showed a higher slope of shear thinning behavior compared to the solutions with lower concentration as seen in Figure 4. Accordingly, the flow behavior index $\mathrm{n}$ decreases as polymer concentration increases (see Table 3) and vice versa for the consistency index $\mathrm{K}$. This is due to the high degree of entanglements present in concentrated solutions. Hence polymer molecules are more sensitive to imposed shear rate that reduces the degree of entanglements resulting in lower viscosity with increasing flow rates [73]. 
Shear viscosity increases with increasing polymer molecular weight or concentration. For a given polymer concentration of $1000 \mathrm{ppm}$, the shear viscosity of polymer $\mathrm{A}, \mathrm{B}$ and $\mathrm{C}$ were 8.4, 13.6 and $19.0 \mathrm{cP}$, respectively, measured at $\dot{\gamma}=10 \mathrm{~s}^{-1}$. We are using a shear rate of $10 \mathrm{~s}^{-1}$ in this study as a reference of reservoir relevant shear rate. The increase of viscosity with $\mathrm{Mw}$ is ascribed to increase in hydrodynamic volume and charge density per molecule. On the other hand, the increase of viscosity with respect to concentration is ascribed to the increase of the number of molecules that increases the interaction and repulsion forces among negatively charged polymer molecules [6].

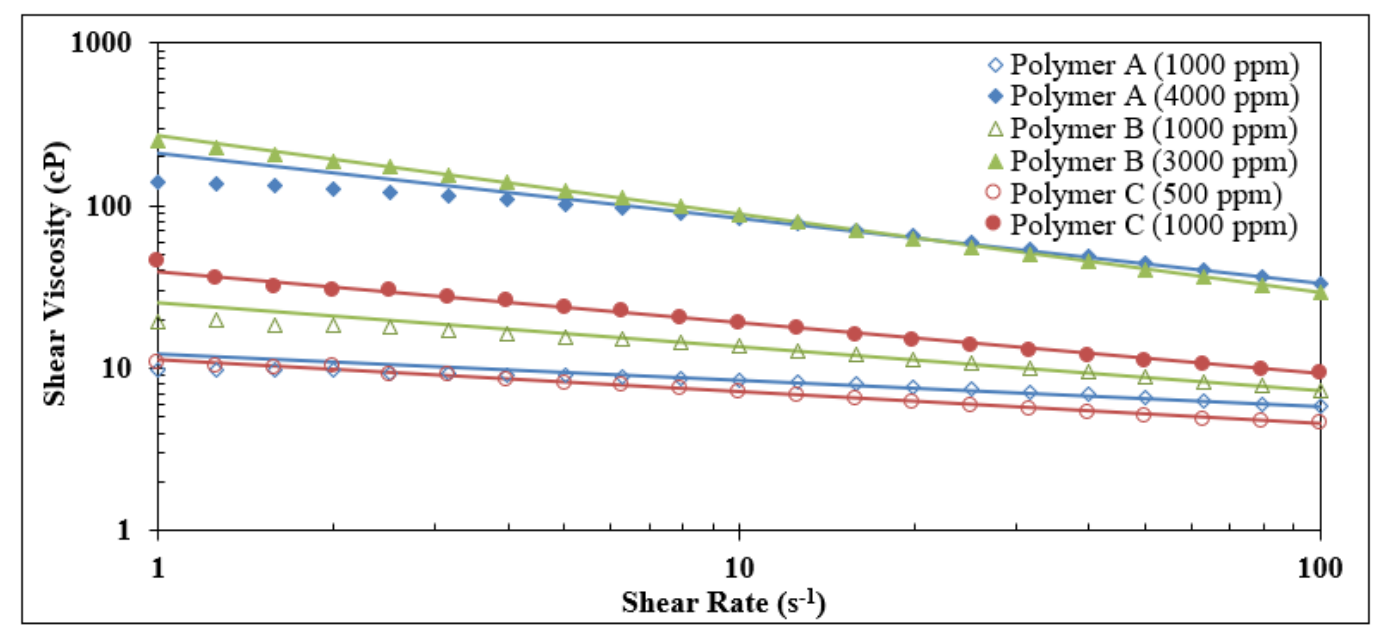

Figure 4. Shear viscosity of prefiltered bulk solutions A, B and $\mathrm{C}$ in $1 \mathrm{wt}$. $\% \mathrm{NaCl}$ at $22{ }^{\circ} \mathrm{C}$. Solid lines represent the power law model.

Table 3. Power law fitting parameters $K, n$ and coefficient of determination $R^{2}$.

\begin{tabular}{ccccccc}
\hline Polymer & \multicolumn{2}{c}{ A } & \multicolumn{3}{c}{ B } & \multicolumn{3}{c}{ C } \\
\hline Concentration (ppm) & 1000 & 4000 & 1000 & 3000 & 500 & 1000 \\
K (cP.s $\left.{ }^{(n-1)}\right)$ & 12.30 & 207.98 & 25.17 & 268.52 & 11.17 & 38.99 \\
$\mathrm{n}$ & 0.84 & 0.60 & 0.73 & 0.52 & 0.80 & 0.69 \\
$\mathrm{R}^{2}$ & 0.93 & 0.95 & 0.97 & 1.00 & 0.99 & 0.98 \\
\hline
\end{tabular}

\subsection{In-Situ Polymer Rheology}

\subsubsection{Apparent Viscosity in Porous Medium vs Bulk Viscosity in Rheometer}

In this study, polymer apparent viscosity in the porous medium is represented by resistance factor (RF) as a function of interstitial velocity. However, translating polymer flow velocity in porous medium to shear rate is required to correlate flow velocity in porous medium with the shear rate in rheometer. Determining the shear rate in porous medium is challenging due to many factors such as wide pore size distributions, tortuosity and complexity of porous media. A conventional formula given in Equation (4) was used to estimate reservoir formation shear rate. Figure 5 shows the viscosity profiles of polymer B (3000 ppm) in porous medium versus bulk shear viscosity in the rheometer. Resistance factor and apparent viscosity profiles of polymer in porous media were consistent. The polymer exhibited a predominantly shear thinning behavior in rheometer while it exhibited different flow behaviors in porous media. At shear rates $\dot{\gamma}<30 \mathrm{~s}^{-1}$, the polymer exhibited shear thinning behavior in porous media while shear thickening behavior was observed at shear rates $\dot{\gamma}>52 \mathrm{~s}^{-1}$. A near-Newtonian behavior was observed during the transition between shear thinning to shear thickening behaviors. Both in-situ behaviors (near-Newtonian and shear thickening) in porous medium were not predicted by shear rheology. This is expected due to the different nature of flow exists in porous medium which is not purely shear flow as in rheometer [37]. 
Figure 5 shows bulk shear viscosity decreased while the apparent viscosity increased. For example, at $\dot{\gamma} \approx 400 \mathrm{~s}^{-1}$, bulk shear viscosity was $16.7 \mathrm{cP}$ while apparent viscosity was $\sim 300 \mathrm{cP}$ which is more than 10 times higher than bulk shear viscosity. This indicates the contribution of extensional viscosity to apparent viscosity at high flow rates. Polymer apparent viscosity is a combination of shear and extensional viscosity of viscoelastic polymers [1].

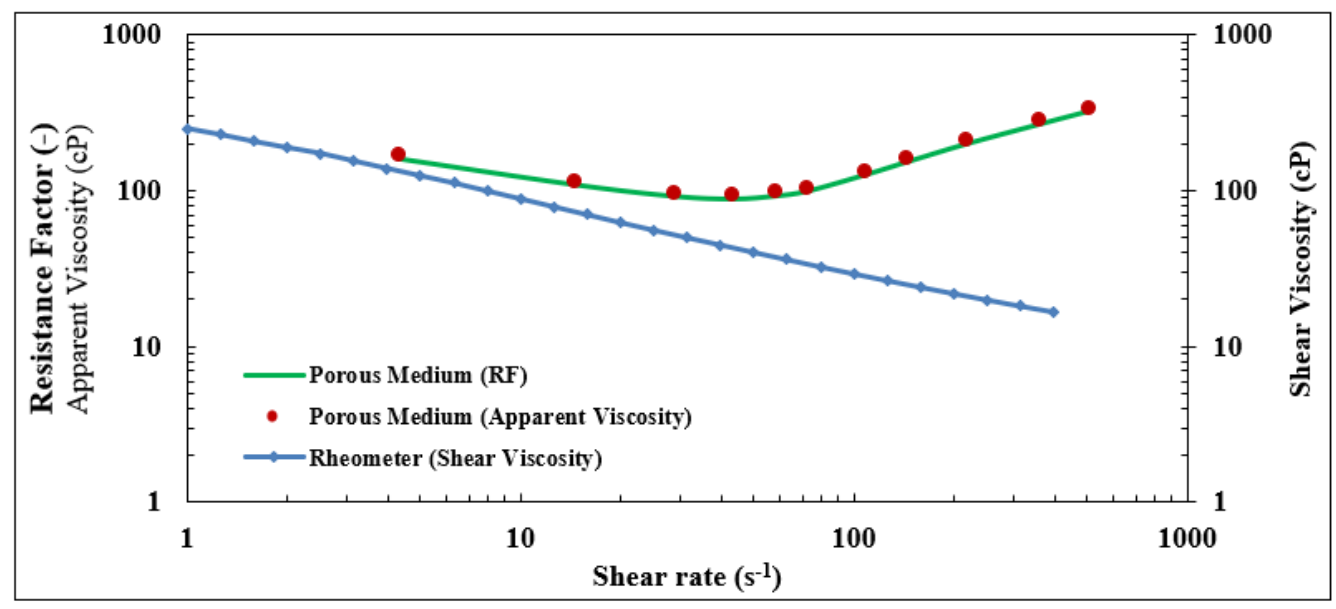

Figure 5. Viscosity profiles of prefiltered Polymer B (3000 ppm) as measured in the porous medium and in bulk.

\subsubsection{Flow of Semi-Dilute Polymer Solutions}

Figure 6 depicts the resistance factor of polymer A, B and C versus interstitial velocity. The concentration of polymer A and B was $1000 \mathrm{ppm}$ while for polymer $C$ was $500 \mathrm{ppm}$. Shear viscosity data of prefiltered solutions $\mathrm{A}, \mathrm{B}$ and $\mathrm{C}$ were $8.4,13.5$ and $7.1 \mathrm{cP}$, respectively, as tabulated in Table 4. At low velocities all the polymers exhibited near-Newtonian behavior followed by shear thickening at high velocities. This represents the general behavior of polymer flow in porous medium for semi-dilute solutions. Similar observations have been reported elsewhere $[11,12,49,50,74]$. The RF curves are strongly dependent on polymer molecular weight. For example, RF at reservoir velocities of polymer A was 2 times lower than that of polymer B which was 18.4. Similarly, shear thickening behavior was more dramatic for polymer $C$ with high $\mathrm{Mw}$. This could be observed from the earlier onset of shear thickening for polymer $\mathrm{C}\left(\mathrm{v}_{\mathrm{c}}=2.5 \mathrm{~m} /\right.$ day) to that of polymers $\mathrm{B}$ and $\mathrm{A}\left(\mathrm{v}_{\mathrm{c}}=4.1\right.$ and $7.0 \mathrm{~m} /$ day, respectively). Moreover, the stronger viscoelastic properties of high $\mathrm{Mw}$ polymer $\mathrm{C}$ can be observed from the slope of apparent shear thickening $7.7(\mathrm{~m} / \text { day })^{-1}$ compared to 3.5 and 0.5 $(\mathrm{m} / \text { day })^{-1}$ for polymer $B$ and $A$, respectively. This yields higher $R F$ values for polymer $C(R F \sim 196)$ at high flow rate compared to that of lower Mw polymers.

It is worth noting that effluents shear viscosity of prefiltered solutions for polymers A and B did not show significant mechanical degradation at the investigated flow velocities. However, prefiltered polymer $\mathrm{C}$ showed degradation at high velocities $(\mathrm{Deg}=21.7 \%$ ). This could be the reason for lower RF values for reinjected polymer $C$ in Figure $6 c$.

Reinjection process was carried out to simulate the polymer flow deep in the reservoir (radially distant from the wellbore). This process also demonstrates the effect of exposure time at high shear on polymer degradation. In this process, the polymer solution passed two cores at different flow rates before measuring in-situ rheology in the main core. The first core (5 $\mathrm{cm}$ length) was used for the pre-filtering process at low flow rate $(Q=0.5 \mathrm{cc} / \mathrm{min})$, while the second core $(10 \mathrm{~cm}$ length) was used as shearing media. Hence, this process differs from the presheared polymer process in which the polymer solution was sheared in a short core and at high flow velocity before the measurement of in-situ rheology in the main core (see Figure 3 for details). Therefore, the results will be compared with preshearing process. Pretreatment methods (reinjection and preshearing) caused a reduction 
of RF values at high velocities while RF values were similar to prefiltered solution at low velocities. This could be clearly seen from the shift of the onset to higher velocities and reduction on the degree of shear thickening. In Figure 6a, RF profile of reinjected polymer A indicates more degradation occurred compared to presheared solution. This is analogous to the observation shown in Figure $6 \mathrm{c}$ for polymer $\mathrm{C}$ at low concentration. This confirms the occurrence of successive degradation as the polymer was reinjected in porous media which is inline with some other studies [68,75]. However, this was not observed for polymer B. The successive polymer degradation observed in this study in contrast to the current understanding of mechanical degradation which is mainly confined to sand face degradation and is believed to be independent of travelled distance in porous media $[38,49,66]$.

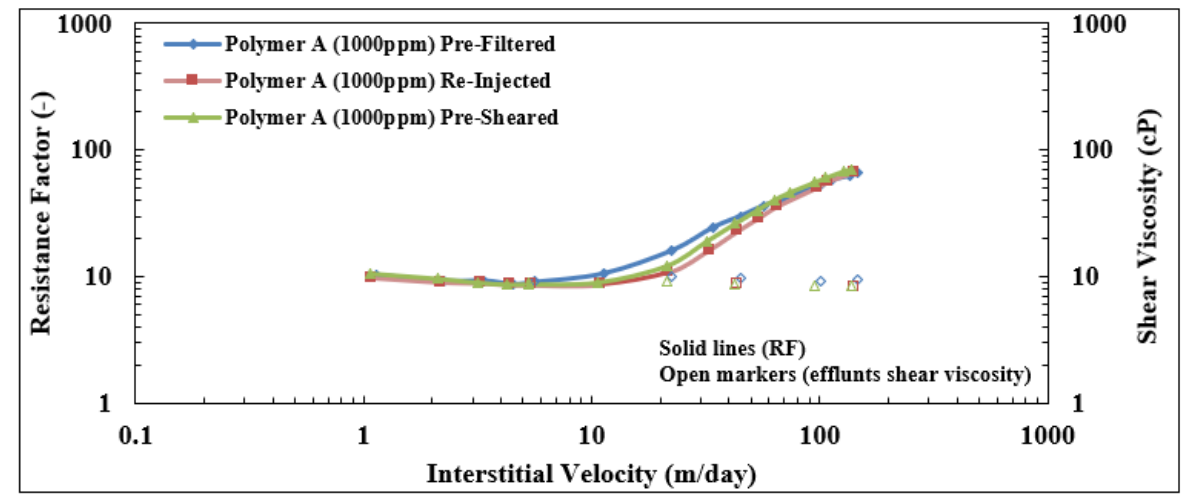

(a)

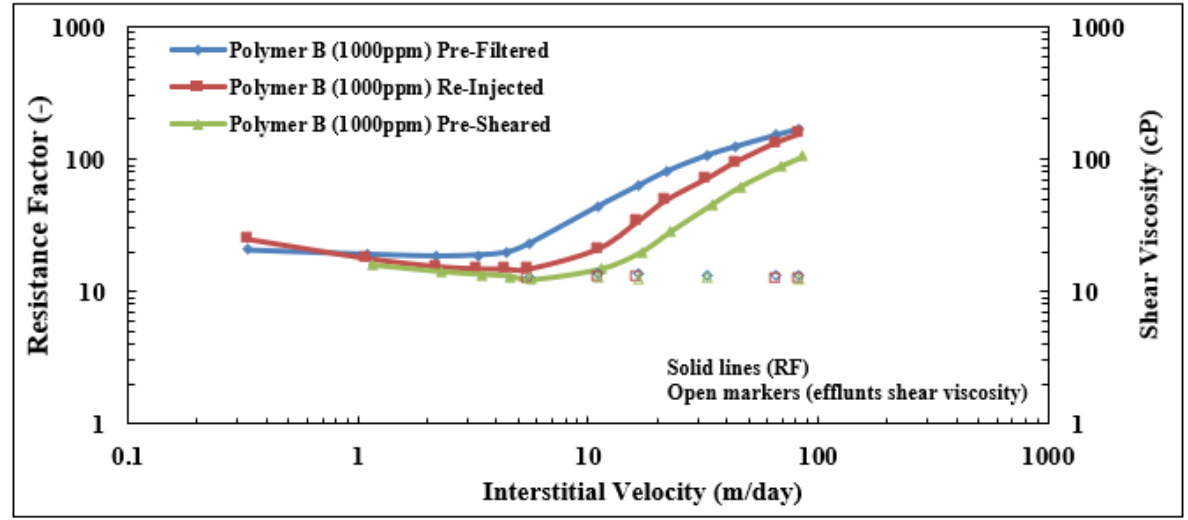

(b)

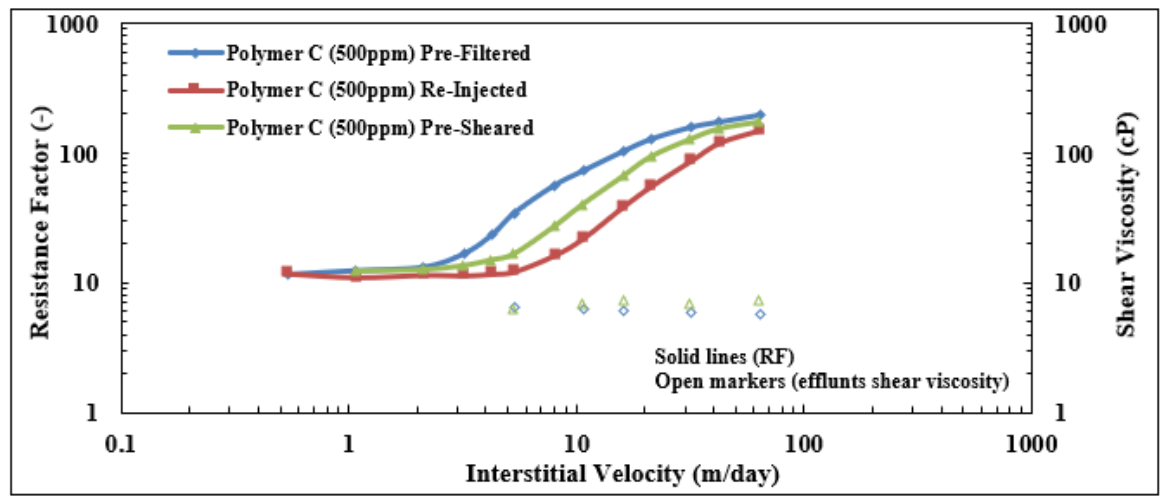

(c)

Figure 6. Resistance factor versus interstitial velocity for semi-dilute polymers A, B and C. Open markers indicate effluent shear viscosity for the given velocity measured at $\dot{\gamma}=10 \mathrm{~s}^{-1}$. (a) Polymer $\mathrm{A}(\mathrm{Mw} \approx 8 \mathrm{MDa}$, Concentration = $1000 \mathrm{ppm})$; (b) Polymer B (Mw $\approx 12 \mathrm{MDa}$, Concentration $=1000 \mathrm{ppm})$; (c) Polymer $\mathrm{C}(\mathrm{Mw} \approx 18 \mathrm{MDa}$, Concentration = $500 \mathrm{ppm})$. 
The difference between shear viscosity of effluents and resistance factor values at low velocities was different depending on polymer type. This indicates that the difference in polymer retention correlates with the increase of polymer molecular weight. That is, the difference between RF values and effluent viscosity at similar velocities increases with the increase of polymer molecular weight. For instance, Newtonian RF values were $\sim 9,18,12$ with bulk shear viscosity of $8.4,13.5$ and $7.1 \mathrm{cP}$ for prefiltered polymers $\mathrm{A}, \mathrm{B}$ and $\mathrm{C}$, respectively.

Table 4. Cores and viscoelastic properties of semi-dilute polymers at concentration of $1000 \mathrm{ppm}$ for polymers $\mathrm{A}$ and $\mathrm{B}$, and 500 ppm for polymer C.

\begin{tabular}{|c|c|c|c|c|c|c|c|c|c|c|}
\hline Experiment & $\begin{array}{c}\mathrm{L} \\
(\mathrm{cm})\end{array}$ & $\begin{array}{c}D \\
(\mathrm{~cm})\end{array}$ & $\begin{array}{c}\phi \\
(-)\end{array}$ & $\underset{\text { (Darcy) }}{\mathrm{K}_{\mathrm{wi}}}$ & $\begin{array}{c}\mathrm{K}_{\mathrm{wf}} \\
\text { (Darcy) }\end{array}$ & $\begin{array}{c}\text { RRF } \\
(-)\end{array}$ & $\underset{(\mathrm{cP})}{\eta_{\mathrm{i}}}$ & $\begin{array}{c}\eta_{\mathrm{e}} \\
(\mathrm{cP})\end{array}$ & $\begin{array}{c}v_{c} \\
(\mathrm{~m} / \text { day })\end{array}$ & $\underset{(m / \text { day })^{-1}}{m}$ \\
\hline Polymer A Pre-Filtered & 9.28 & 3.79 & 0.23 & 1.81 & 1.12 & 1.61 & 8.44 & 8.19 & 6.98 & 0.54 \\
\hline Polymer A Re-Injected & 9.74 & 3.79 & 0.23 & 2.26 & 1.16 & 1.95 & 8.19 & 8.28 & 16.92 & 0.53 \\
\hline Polymer A Pre-Sheared & 10.22 & 3.77 & 0.24 & 2.40 & 1.48 & 1.62 & 8.52 & 8.42 & 15.79 & 0.66 \\
\hline Polymer B Pre-Filtered & 9.82 & 3.79 & 0.23 & 2.16 & 0.96 & 2.24 & 13.57 & 13.31 & 4.06 & 3.50 \\
\hline Polymer B Re-Injected & 9.57 & 3.79 & 0.23 & 2.08 & 1.24 & 1.68 & 13.31 & 12.75 & 7.69 & 2.28 \\
\hline Polymer B Pre-Sheared & 10.27 & 3.77 & 0.23 & 2.80 & 1.54 & 1.82 & 13.54 & 12.75 & 11.99 & 1.46 \\
\hline Polymer C Pre-Filtered & 9.82 & 3.77 & 0.24 & 2.57 & 1.40 & 1.84 & 7.11 & 5.79 & 2.51 & 7.68 \\
\hline Polymer C Re-Injected & 9.78 & 3.77 & 0.24 & 2.39 & 1.28 & 1.86 & 5.79 & - & 6.71 & 3.00 \\
\hline Polymer C Pre-Sheared & 9.72 & 3.77 & 0.24 & 2.25 & 0.82 & 2.75 & 7.13 & 7.21 & 4.00 & 4.32 \\
\hline
\end{tabular}

\subsubsection{Flow of Concentrated Polymer Solutions}

The behavior of concentrated polymer solutions $\left(C>>C^{*}\right)$ in porous media seems to be dominated by shear viscosity that overrides their elastic properties. In such solutions, the interaction between polymer molecules is dominant and polymer chains are entangled [11]. Figure 7 shows apparent shear thinning, near-Newtonian and shear thickening behaviors which become very important flow aspects for high concentrated polymer solutions with high shear viscosity. For instance, Figure 7a depicts the flow of polymer A at concentration of $4000 \mathrm{ppm}$ in porous media, in particular parlance, it exhibits pseudo-gel behavior. As this figure shows for prefiltered polymer $\mathrm{A}$, at high velocities ( $\mathrm{v}>31.0 \mathrm{~m} /$ day) weak shear thickening behavior is observed with slight increase in RF values compared to near-Newtonian plateau at flow velocities $5.7<\mathrm{v}<31.0 \mathrm{~m} /$ day. RF values corresponding to shear thickening behavior are lower than shear viscosity. This suggests that the contribution of extensional viscosity is lower than that of shear viscosity which dominates the polymer flow behavior. Below $\mathrm{v}<5.7 \mathrm{~m} /$ day, the RF values increase with decreasing flow velocity indicating strong shear thinning behavior. Similar trend was observed for relatively higher Mw polymer B except that the shear thickening becomes stronger (see Figure $7 \mathrm{~b}$ ). This confirms that the concentration or in other words, solution conformational regime, has an obvious influence on polymer in-situ rheology. However, concentrated high Mw polymer C (Figure 7c) exhibits near-Newtonian behavior at lower shear rates that are analogous to semi-dilute polymers discussed earlier without the presence of shear thinning behavior. This could be ascribed to lower shear viscosity of polymer $\mathrm{C}$ at $1000 \mathrm{ppm}$ compared to the other two polymers A and B. Higher molecular weight polymers possess higher RF at high velocity than the low Mw polymers with higher concentration. This also indicates the contribution of higher elastic properties such as elastic viscosity compared to that of lower Mw solutions. 


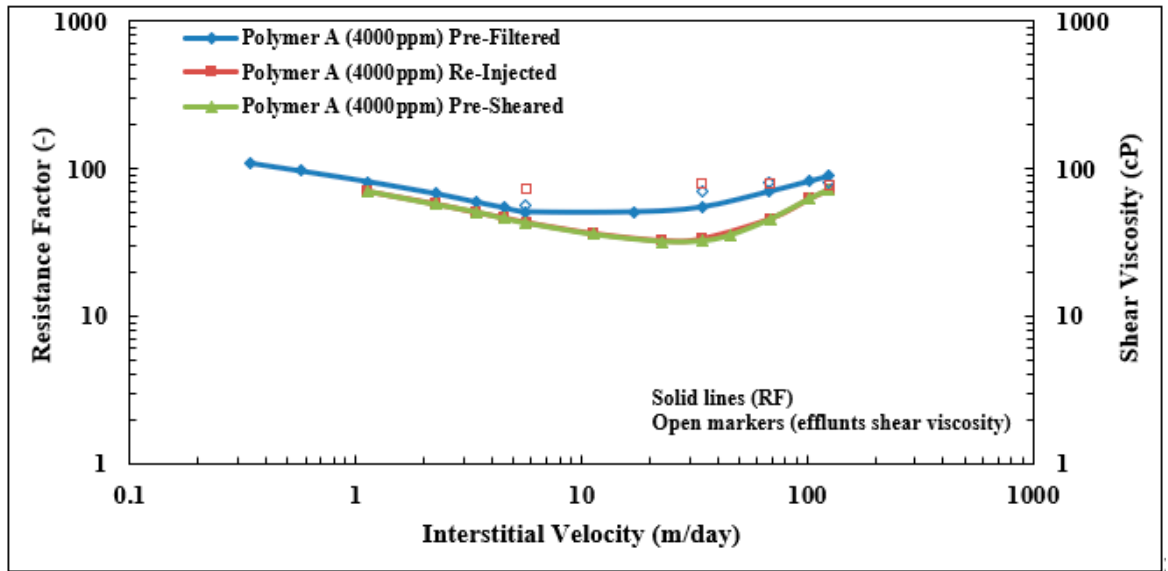

(a)

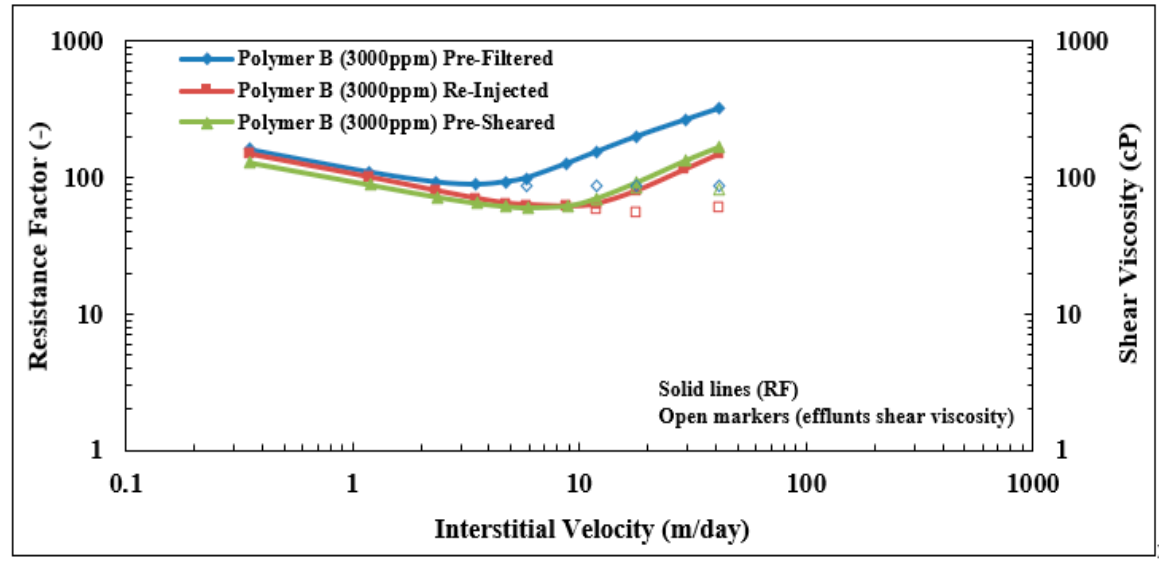

(b)

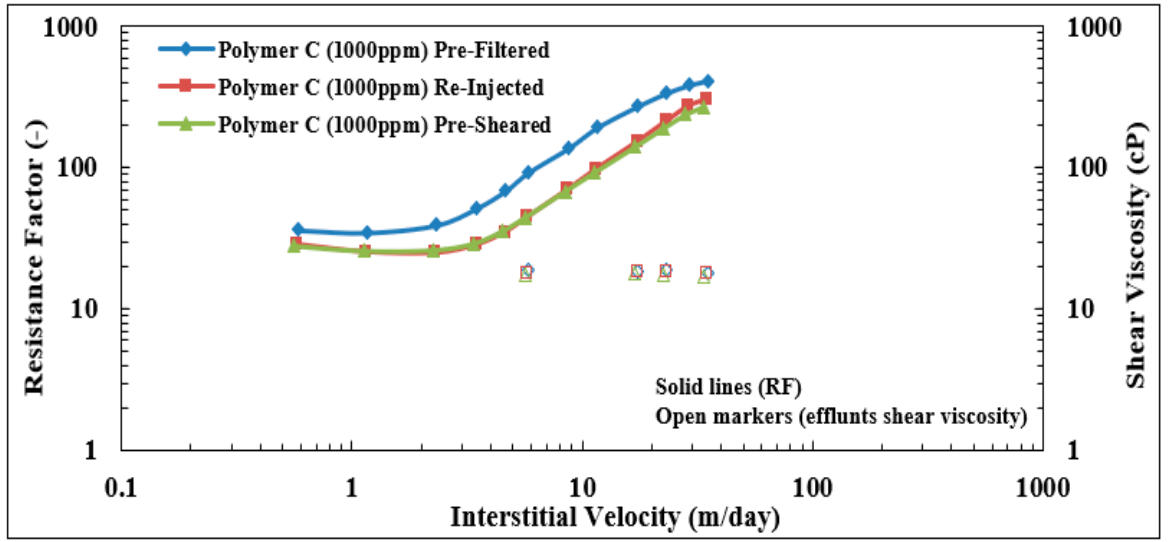

(c)

Figure 7. Resistance factor versus interstitial velocity for concentrated polymers A B and C at concentration of 40003000 and 1000 ppm, respectively. Open markers indicate effluent shear viscosity for the given velocity measured at $\dot{\gamma}=10 \mathrm{~s}^{-1}$. (a) Polymer $\mathrm{A}(\mathrm{Mw} \approx 8 \mathrm{MDa}$, Concentration $=4000$ ppm); (b) Polymer B $(\mathrm{Mw} \approx 12 \mathrm{MDa}$, Concentration = $3000 \mathrm{ppm})$; (c) Polymer C $(\mathrm{Mw} \approx 18 \mathrm{MDa}$, Concentration $=1000 \mathrm{ppm})$.

The existence of shear thinning in porous media is conditional and argued by Seright et al. [76] at which they attribute shear thinning to the presence of micro-gels in polymer solution. However, the results in Figure 7 show a contradictory observation and confirm the existence of shear thinning 
phenomena for concentrated solution in porous media even if the polymer has been pre-filtered or presheared (microgel-free).

Exposing the polymer to wellbore mechanical degradation alters its viscoelastic behavior through the shift of Newtonian plateau and onset of shear thickening to higher velocities as is seen in Figure 7 for both reinjected and presheared solutions A and B. However, insignificant alteration occurs on shear thinning part. Effluent viscosity of reinjected solution B suffers more degradation compared to presheared sample which could be due to attachment of backpressure device that might induce additional degradation to polymer solution [67]. RF curves of degraded solutions C (reinjected and presheared) are coincident which indicates that the solution tolerates wellbore mechanical degradation compared to semi-dilute solution with same polymer $C$ discussed above (see Figure $6 c$ ). However, the RF curves of degraded solution $C$ were lower than prefiltered solution which could be due to filtration effect and also lower RRF (see Table 5).

Table 5. Cores and viscoelastic properties of concentrated polymers A, B and C at concentration of 4000, 3000 and 1000 ppm, respectively.

\begin{tabular}{|c|c|c|c|c|c|c|c|c|c|c|}
\hline Experiment & $\begin{array}{c}\mathrm{L} \\
(\mathrm{cm})\end{array}$ & $\begin{array}{c}D \\
(\mathrm{~cm})\end{array}$ & $\begin{array}{c}\phi \\
(-)\end{array}$ & $\begin{array}{c}\mathbf{K}_{\text {wi }} \\
\text { (Darcy) }\end{array}$ & $\begin{array}{c}\mathbf{K}_{\mathrm{wf}} \\
\text { (Darcy) }\end{array}$ & $\underset{(-)}{\mathrm{RRF}}$ & $\begin{array}{c}\eta_{i} \\
(\mathrm{cP})\end{array}$ & $\begin{array}{c}\eta_{\mathrm{e}} \\
(\mathrm{cP})\end{array}$ & $\begin{array}{c}v_{c} \\
\text { (m/day) }\end{array}$ & $\underset{(\mathrm{m} / \text { day })^{-1}}{\mathrm{~m}}$ \\
\hline Polymer A Pre-Filtered & 9.69 & 3.77 & 0.23 & 2.75 & 1.48 & 1.86 & 83.02 & 79.31 & 31.00 & 0.37 \\
\hline Polymer A Re-Injected & 10.05 & 3.74 & 0.23 & 2.53 & 1.58 & 1.60 & 79.31 & 76.74 & 43.00 & 0.43 \\
\hline Polymer A Pre-Sheared & 9.95 & 3.77 & 0.23 & 2.50 & 1.84 & 1.36 & 77.91 & - & 42.00 & 0.45 \\
\hline Polymer B Pre-Filtered & 9.85 & 3.79 & 0.22 & 2.40 & 0.73 & 3.31 & 88.76 & 85.90 & 4.37 & 8.45 \\
\hline Polymer B Re-Injected & 9.52 & 3.78 & 0.22 & 2.64 & 1.04 & 2.53 & 85.90 & 66.12 & 11.21 & 2.96 \\
\hline Polymer B Pre-Sheared & 9.93 & 3.78 & 0.22 & 2.35 & 0.85 & 2.77 & 83.79 & 80.79 & 9.08 & 3.59 \\
\hline Polymer C Pre-Filtered & 10.04 & 3.78 & 0.22 & 2.12 & 0.23 & 9.27 & 18.95 & 17.98 & 2.31 & 16.07 \\
\hline Polymer C Re-Injected & 9.81 & 3.78 & 0.22 & 2.01 & 0.33 & 6.09 & 17.98 & 17.46 & 3.78 & 9.71 \\
\hline Polymer C Pre-Sheared & 9.68 & 3.78 & 0.23 & 2.37 & 0.80 & 2.96 & 17.86 & 17.14 & 3.40 & 8.47 \\
\hline
\end{tabular}

\subsubsection{Onset and Slope of Shear Thickening Behavior}

Figure 8 presents the resistance factor change for prefiltered polymers $\mathrm{A}, \mathrm{B}$ and $\mathrm{C}$ at different concentrations. As this figure shows, RF is influenced by both polymer molecular weight and concentration. That is, RF gains strength with increasing Mw and concentration. This is inline with the increase in shear viscosity shown in Figure 4 where shear viscosity increases with increase in $\mathrm{Mw}$ and concentration. However, the impact of $\mathrm{Mw}$ and concentration on RF values in the porous media is a function of velocity. RF is dominated by molecular weight at high velocities to a greater extent than concentration and vice versa at low velocities below the onset of shear thickening. For instance, RF curves of polymer A converge to similar values at high velocities regardless of the significant difference in concentration while the concentration differentiates the RF at low velocities. This indicates the contribution of both shear and elastic viscosity in polymer flow through porous media, although shear viscosity reaches its minimum at high velocities [74]. The degree and magnitude of shear thickening increase with the increase in molecular weight and concentration. This highlights the influence of shear and elastic viscosities on the slope of apparent shear thickening [37].

The onset of shear thickening and the flow behavior of polymer are more important than the extensional magnitude itself in determining the suitability of polymer for EOR applications [77]. Hence, onset of shear thickening has received extensive attention in the literature $[50,52,53,78]$. Figure 8 shows polymer molecular weight has an obvious influence on the onset of shear thickening. A solution with higher molecular weight experiences earlier onset of shear thickening. With increasing polymer $\mathrm{Mw}$, the apparent shear thickening increases, conversely, the onset of shear thickening shifts to lower velocities [79].

Moreover, Figure 8 shows that onset of shear thickening is independent of polymer concentration. This observation excludes 4000 ppm polymer A which exhibits a gel-like behavior and yields shift of the onset to higher velocities. This confirms that the conformational state of polymer solution has more influence on the onset of shear thickening compared to the concentration value itself. This could be one of the reasons that cause a controversial observations in the literature regarding the correlation 
between the onset and polymer concentration. For example, Chauveteau and Moan [52] reported the onset of shear thickening decreases with increasing polymer concentration for investigated wide concentration range (21-1360 ppm). However, a close look on the reported data, we could see the onset was almost similar for a specific concentration range (e.g., 170-680 ppm). This again indicates the conformational state influences the onset of shear thickening. For instance, within a semi-dilute regime, the onset of shear thickening decreases with molecular weight increases regardless of concentration $[79,80]$. The apparent viscosity from the resistance factor gradually increases as polymer concentration increases [81].

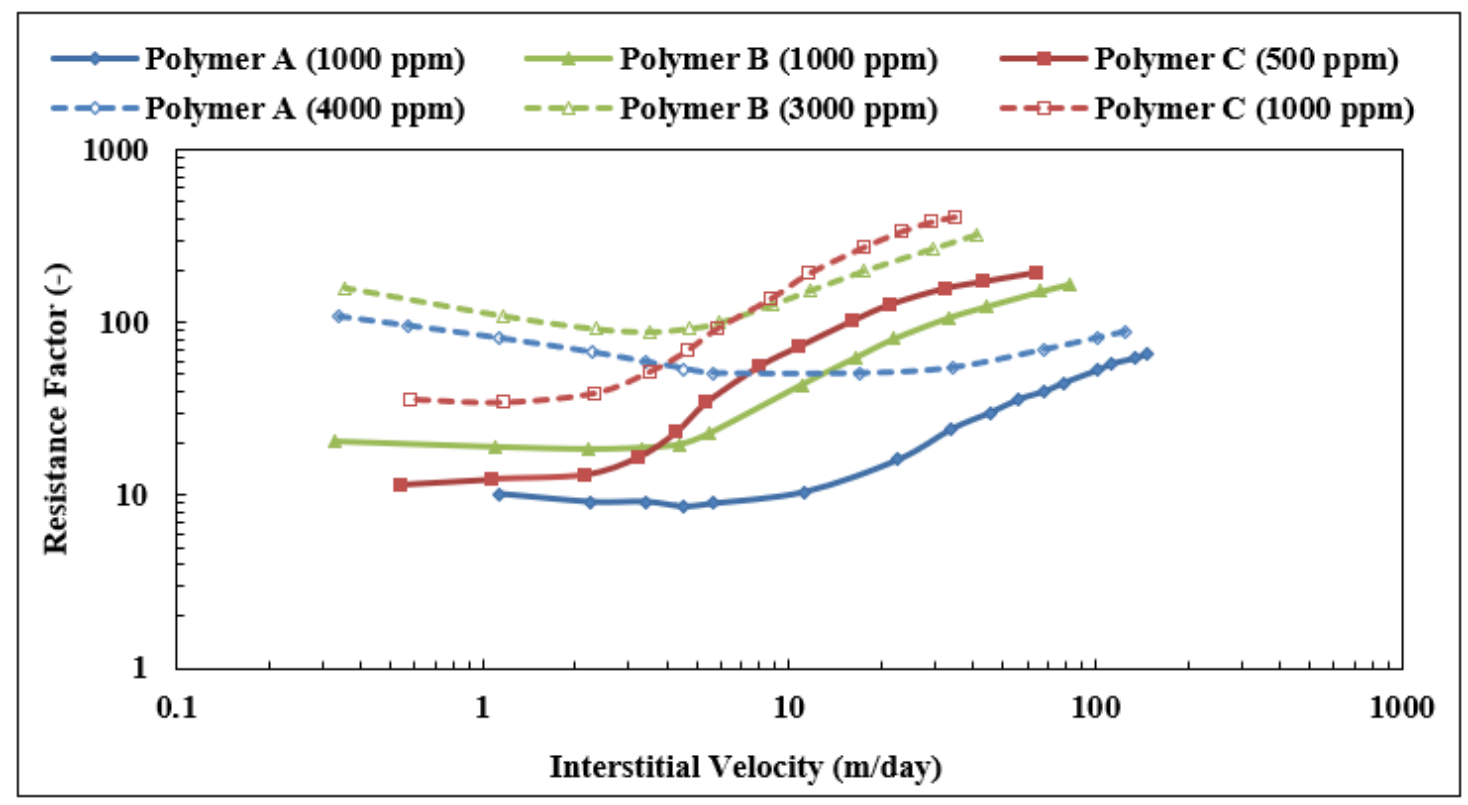

Figure 8. Resistance factor versus interstitial velocity for prefiltered polymers A, B and C at different concentrations.

\subsection{Polymer Mechanical Degradation}

The presented results showed the impact of wellbore mechanical degradation on both bulk shear viscosity and in-situ rheology. Despite the insignificant impact of mechanical degradation on shear viscosity of effluents, a considerable alteration of in-situ rheology behavior occurred. The significant alteration was found with reduction of apparent shear thickening behavior by shifting its onset to higher velocity and reduction of the slope while maintaining in-situ viscosity. The amount of alteration was influenced by polymer conformational regime. For instance, the change of the onset of shear thickening by comparing presheared solution A with a prefiltered sample was $126.2 \%$ at concentration of $1000 \mathrm{ppm}$, while this percentage drops to $35.5 \%$ when polymer concentration increases to $4000 \mathrm{ppm}$. This is also valid for polymers B and C. This elucidates that increasing polymer concentration is beneficial for polymer shear stability [36,82]. However, the impact of mechanical degradation on the slope of shear thickening was independent of polymer conformation regime (concentration). For example, the change of the slope of shear thickening of presheared solution B compared to prefiltered sample was $58.6 \%$ at concentration of 1000 ppm while it was 57.5\% when polymer concentration increased to $3000 \mathrm{ppm}$. Similar observations were found for the other two polymers. This indicates that the impact of wellbore mechanical degradation on shifting onset of shear thickening to higher velocities was lower for concentrated solutions compared to that of semi-dilute polymer solutions. The change in the slope of shear thickening due to mechanical degradation seems independent of concentration. 


\subsubsection{Influence of Mechanical Degradation on In-Situ Rheology}

Figure 9 compares the impact of mechanical degradation on the reduction of RF values of high Mw polymers B and C to RF of prefiltered polymer A which has relatively lower Mw. Recall from the discussion above, polymer $\mathrm{Mw}$ is a dominating factor on the polymer flow behavior after onset of shear thickening for semi-dilute polymers. Reduction of slope and shift of onset of shear thickening to higher velocities is an indication of a reduction of polymer MWD [50]. For example, the degree was reduced and onset of shear thickening of high $\mathrm{Mw}$ Polymer $\mathrm{C}(\mathrm{Mw} \approx 18 \mathrm{MDa})$ shifted to higher velocities due to preshearing. Therefore, RF curve similar to that of lower Mw prefiltered Polymer $\mathrm{B}(\mathrm{Mw} \approx 12 \mathrm{MDa}$ ) was achieved. A similar observation was found for presheared polymer $B$ where preshearing resulted in shifting $\mathrm{RF}$ values closer to $\mathrm{RF}$ of prefiltered polymer $\mathrm{A}(\mathrm{Mw} \approx 8 \mathrm{MDa})$. This was also observed for concentrated solutions as shown on the right Figure 9.



(a)

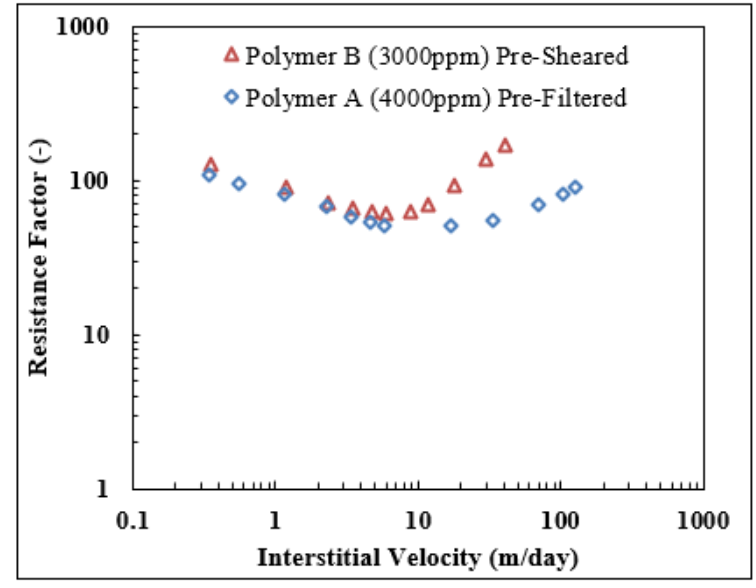

(b)

Figure 9. Influence of preshearing on degrading the resistance factor of high Mw polymers to that of lower Mw prefiltered solutions. (a) Semi-dilute polymer solutions; (b) Concentrated polymer solutions.

\subsubsection{Mechanical Degradation at Elevated Velocities}

Figure 10 displays the effect of mechanical degradation on shear viscosity of effluent polymer solutions at a broad range of shear rates. These experiments were designed to compare the degradation effect in different polymers which have been exposed to comparable shear rate. It is clear that polymer $\mathrm{C}$ with high $\mathrm{Mw}$ experienced more shear degradation at similar injection rate applied for all solutions. For instance, degradation at $\mathrm{Q}=90 \mathrm{cc} / \mathrm{min}$ was $4.0 \%, 12.0 \%, 20.0 \%$ for polymers $\mathrm{A}, \mathrm{B}$ and C, respectively. 


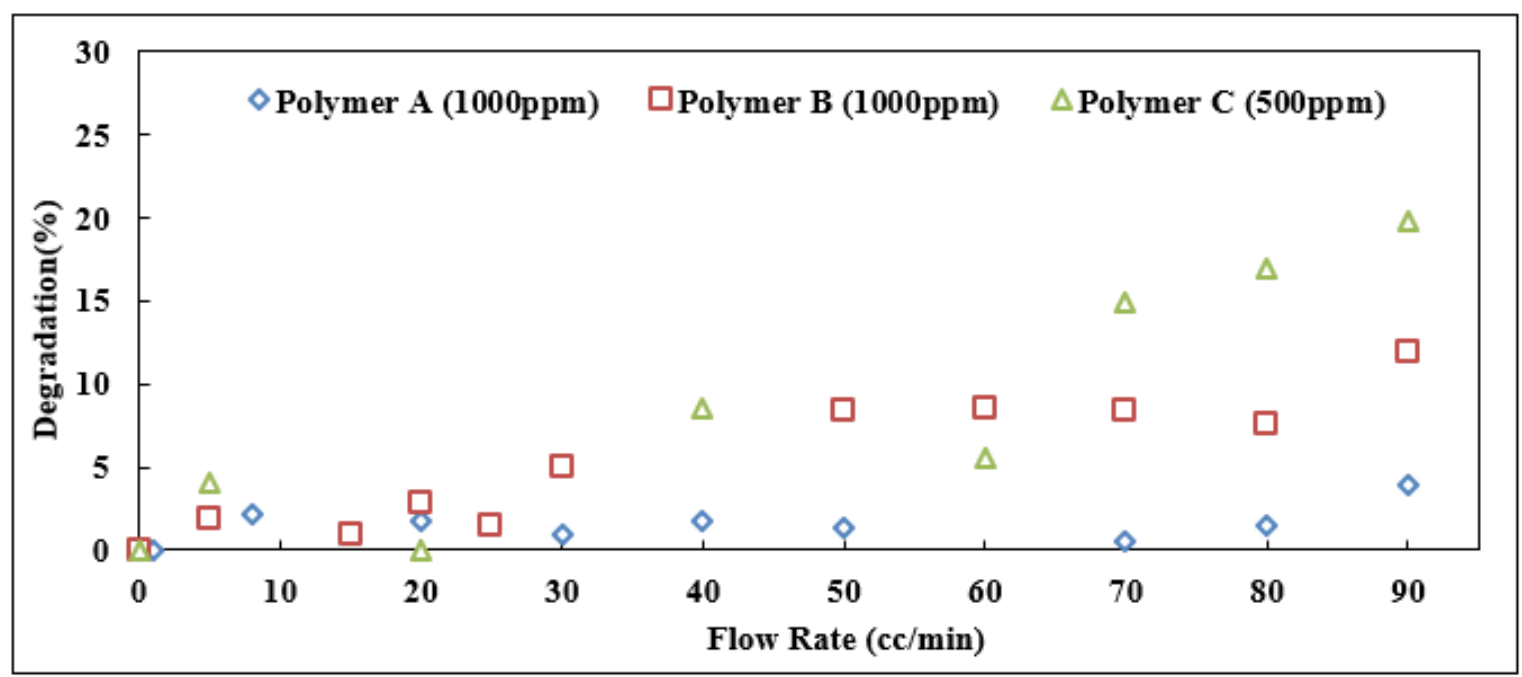

Figure 10. Shear degradation at elevated velocities for polymer A, B and C.

Figure 11 shows the in-situ flow of polymer B that has been presheared at different flow rates. Fresh (undegraded) solution is the same solution described in Figure 10 that has a shear viscosity of $13.3 \mathrm{cP}$ and was injected into short Bentheimer core at various injection rates. It can be seen that the RF profile of fresh solution is identical to RF profile of prefiltered solution at similar velocities. This indicates that prefiltering at low flow rates $\left(\mathrm{Q} \leq 0.5 \mathrm{cc} / \mathrm{min}, v_{\mathrm{D}} \leq 0.6 \mathrm{~m} /\right.$ day $)$ will not alter $\mathrm{RF}$ values. However, increasing preshearing rate to $\left(\mathrm{Q}=15 \mathrm{cc} / \mathrm{min}, v_{\mathrm{D}}=19.4 \mathrm{~m} /\right.$ day) will significantly alter viscoelastic properties such as the onset and degree of shear thickening, while not significantly affecting in-situ viscosity and bulk shear viscosity. However, preshearing the polymer at very high injection rate $\left(\mathrm{Q}=110 \mathrm{cc} / \mathrm{min}, v_{\mathrm{D}}=141.2 \mathrm{~m} /\right.$ day $)$ causes a shear degradation of $16 \%$ and a considerable reduction ( $>50 \%$ ) on in-situ viscosity by comparing its Newtonian plateau that was observed in porous media with prefiltered solution. The reduction of polymer viscoelastic properties such as the onset and degree of shear thickening is extremely high. The maximum RF value of presheared solution at $Q=110 \mathrm{~m} /$ day was 26.6 which is more than 6 times lower than that of prefiltered solution at comparable velocity. Additionally, RRF of presheared solution at $Q=110 \mathrm{cc} / \mathrm{min}$ was reduced to 1.7 compared to 1.8 and 2.2 for presheared solution at $Q=15 \mathrm{cc} / \mathrm{min}$ and prefiltered solution at $\mathrm{Q}=0.5 \mathrm{cc} / \mathrm{min}$, respectively.

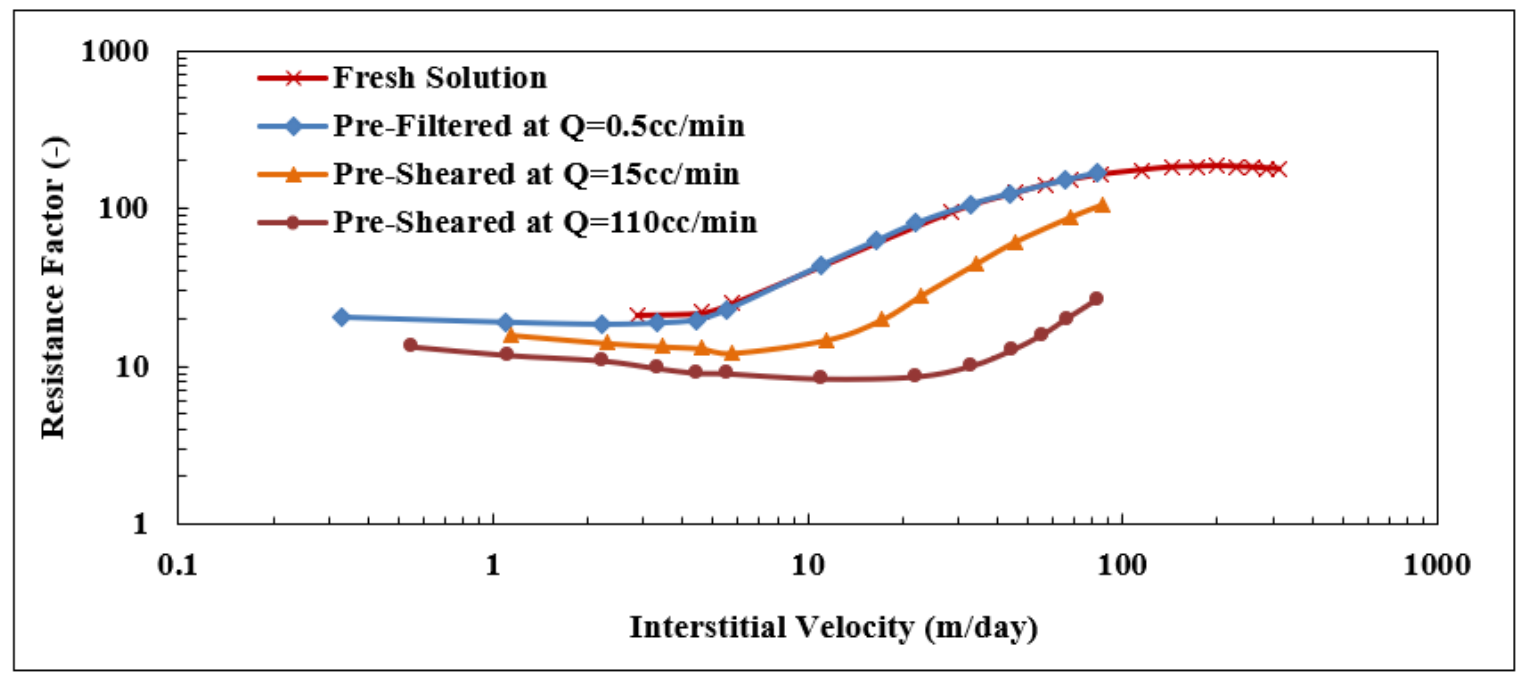

Figure 11. Resistance factor versus interstitial velocity for polymer B presheared at different flow rates. 


\subsection{Permeability Reduction}

Polymer retention in porous media has similar importance to its viscoelasticity (discussed above) on the sweep efficiency and injectivity. It is a determining parameter in screening EOR polymers. When HPAM is transported in porous media, it tends to adsorb on rock surfaces and may trap within small pores resulting in polymer retention. Polymer retention reflects different mechanisms such as adsorption, straining (mechanical entrapment) and hydrodynamic retention (rate effect) [72,83]. As a consequence of polymer retention, permeability reduction occurs. Experimentally, permeability reduction can be evaluated by residual resistance factor (RRF) [57].

$R R F$ is a measure of the extent of permeability reduction of porous media due to polymer injection. RRF correlates directly with the permeability of the porous media and the molecular weight of polymer. Therefore, higher RRF values may result from polymer flooding in low permeability reservoirs using high molecular weight polymers. Generally, for homogeneous porous media with lower contrast in permeability of different zones or layers, the intention is to obtain lower RRF values (RRF $\leq 2$ ) while keeping RF values higher possible at reservoir velocities [84]. This is to increase the sweep efficiency of polymer flooding process by reducing the mobility ratio of displacing and displaced fluids. However, higher RRF values can be beneficial where the porous media is heterogeneous with significant contrast in permeability of different layers. In such cases, higher RRF values could result in better conformance control and thereby improved sweep efficiency through flow diversion into un-swept regions.

Figure 12 depicts RRF values measured for prefiltered polymers A, B and C at different concentrations. It can be seen that concentrated polymers have higher RRF compared to solutions with lower concentration. Furthermore, RRF appears to be significantly dependent on polymer $\mathrm{Mw}$ to a greater extent than concentration. This could be elucidated by looking at a similar polymer concentration of $1000 \mathrm{ppm}$, we can see RRF for polymers A, B and C were 1.6, 2.2 and 9.3 respectively. This emphasizes the increase of RRF with Mw [79].

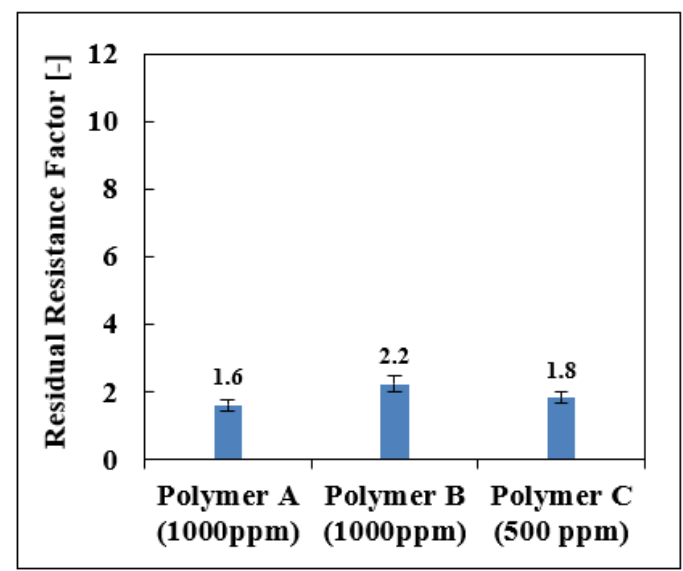

(a)

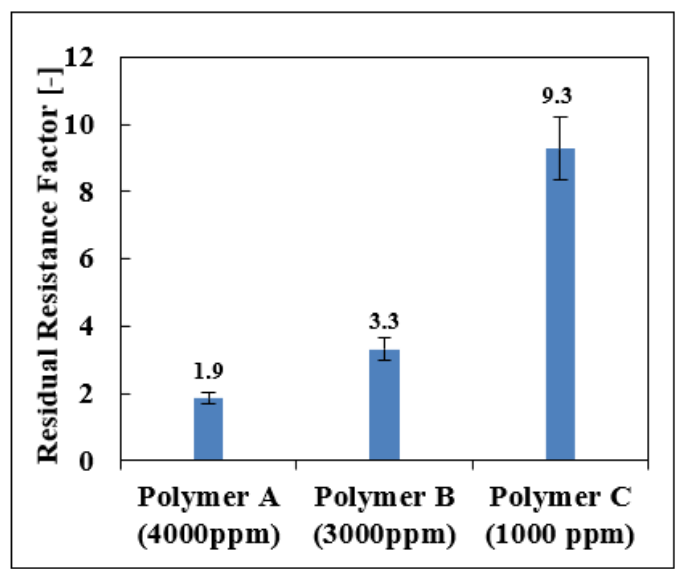

(b)

Figure 12. Residual resistance factor (RRF) of prefiltered polymers A, B and C. (a) Semi-dilute polymer solutions; (b) Concentrated polymer solutions.

Figure 13 displays the impact of polymer pretreatment (prefiltering, reinjecting and preshearing) on RRF values. Presented RRF values are quite scattered. This could be due to challenges on measuring 'true' RRF which has been debated in the literature [84]. One reason could be due to experimental artifacts ascribed to the amount of brine and strategies applied during brine post-flush such as tapering [67]. Another reason could be due to unapproachable steady-state condition during the injection of brine alone after polymer flooding to satisfy Darcy's Law conditions in Equation (2). This is suggested due to the viscoelasticity of retained molecules [85]. However, in some cases, the impact of mechanical degradation on RRF was not significant. This might be due to the reason that high molecular species tend to adsorb first as the polymer transports in porous media. High molecular 
species could also be found in degraded solutions if degradation is not significant which may be enough to form a similar adsorbed layer of non-degraded solutions [70]. Measuring 'true' RRF is an essential task that would certainly improve the estimation of effective polymer viscosity in porous media by using the term RF/RRF.

However, the general trend from the data presented in Figure 13 shows that pretreatment of polymer solutions prior to injection into the porous media results in a reduction in RRF values. That is, RRF values of prefiltered solutions are generally higher than RRF of presheared and reinjected solutions.

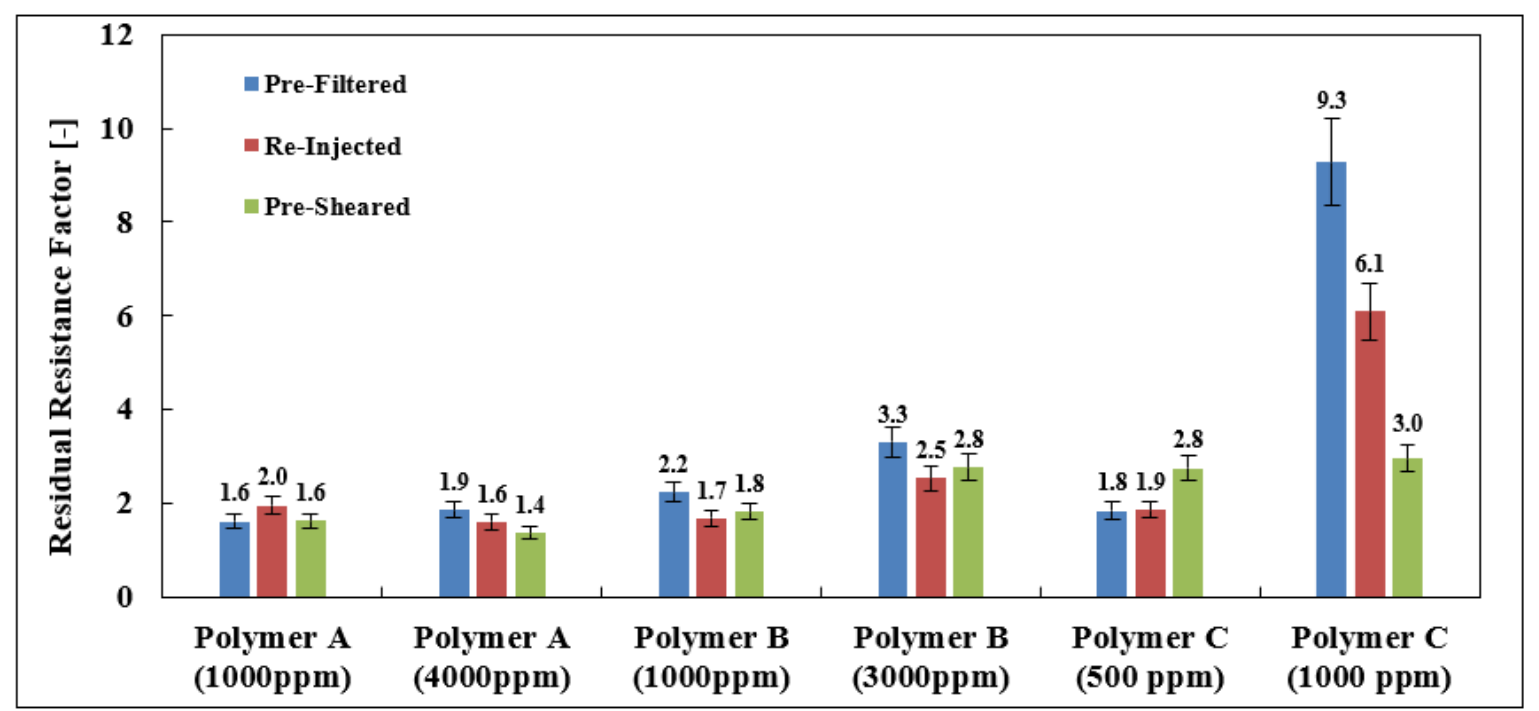

Figure 13. Residual resistance factor (RRF) of polymers A, B and C at different concentrations.

\subsection{Polymer Injectivity}

As stated before, polymer injectivity is a measure of how efficiently polymer solution can be delivered into reservoir matrix. The shear viscosity measurement alone cannot predict injectability of polymer solutions, as it does not reflect the existence of all flow regimes during polymer flow in porous media. Besides other factors, matrix fracturing is one major concern in polymer flooding projects that restricts polymer injection. Injection under matrix condition that may evolve into fracture formation is only a function of injection pressure, irrespective of polymer bulk viscosity [86]. This means fracture initiation is more attributed to polymer viscoelasticity, particularly shear thickening behavior that yields significant pressure build-up. Therefore, polymer injectivity could be inferred from RF and RRF measurements. The pressure gradient associated with high RF values that are found at wellbore region reduces polymer injectivity. Additionally, the decline in polymer injectivity might occur when retention is high as reflected by high RRF in this study (e.g., RRF > 3 is not recommended for EOR applications). Shear thickening may be dampened for each polymer either by increasing the polymer concentration or mechanically degrading the polymer solution before the injection. From economical perspective, the former requires increasing the dosage of polymer which subsequently demands high cost. It may also yield in high RRF. Whereas the latter relates to polymer type more specifically polymer Mw at which the cost of the manufacturing process of low and high Mw polymer is quite similar [87]. The loss of shear viscosity within 20-30\% due to preshearing could be tolerated economically and cause a significant reduction in extensional viscosity that results in a reduction of resistance factor [71]. Additionally, preshearing could also be beneficial, so that high molecular species are sheared and avoided. These species are mainly responsible for wellbore plugging problems that result in permeability reduction. This may increase the shear rates at wellbore area that promotes pressure build-up and eventually mechanically degrade the polymer solution. This suggests 
preshearing high Mw polymer solution is a favorable strategy to optimize its injectivity which is consistent with other studies [88].

\section{Conclusions}

Series of core flood experiments have been performed to investigate in-situ behavior of partially hydrolyzed polyacrylamide polymers (HPAM) in porous media. The influence of HPAM molecular weight and concentration on polymer in-situ rheology has been investigated. Additionally, the impact of mechanical degradation on polymer rheological behavior has been studied. Based on the results, polymer injectivity can be optimized. More specifically, the following conclusions could be made:

- In-situ rheological behavior of HPAM in porous media is different from bulk rheology observed in the rheometer.

- $\quad$ Shear thickening behavior was observed at high velocities representative of those present in the near wellbore region. Near-Newtonian behavior was observed at low velocities representative of those present deep in the reservoir.

- $\quad$ The degree and magnitude of shear thickening increased for higher polymer $\mathrm{Mw}$ and concentration.

- Shear thinning behavior at low velocities was observed for concentrated solutions while not for semi-dilute solutions.

- Exposing HPAM solutions to mechanical degradation through preshearing process prior to injection facilitates its flow in porous media and enhances its injectivity. This is ascribed to a reduction in viscoelastic properties.

- $\quad$ Onset of shear thickening shifted to higher velocities.

- $\quad$ The magnitude and the degree of shear thickening behavior were reduced while in-situ viscosity at low flow rates was maintained.

- $\quad$ RRF appears to be dominated by molecular weight and concentration.

- $\quad$ High RRF found for high molecular weight polymers with high concentration.

- $\quad$ Degraded solutions have lower RRF values specifically for concentrated solutions of high Mw polymers. This effect was more pronounced when polymer solution was degraded at very high velocities.

- Improvement (reduction) in polymer viscoelastic properties and RRF through preshearing process can optimize polymer injectivity.

Author Contributions: Conceptualization, T.S. and A.S.; Formal analysis, B.A.-S., T.S., B.S.S. and A.S.; Investigation, B.A.-S.; Methodology, B.A.-S., and B.S.S.; Project administration, A.S.; Supervision, T.S., B.S.S. and A.S.; Writing—original draft, B.A.-S.; Writing—review \& editing, T.S., B.S.S. and A.S.

Funding: This research received no external funding.

Acknowledgments: We wish to thank Energi Simulation, Canada and the PETROMAKS 2 program at Norwegian Research Council for financial support. We also acknowledge Petroleum Development Oman (PDO) for providing study scholarship for Badar Al-Shakry.

Conflicts of Interest: The authors declare no conflict of interest. 


\section{Nomenclature}

$\mathrm{A}=$ core cross-sectional area $\left(\mathrm{cm}^{2}\right)$

AF4 = Asymmetrical flow field-flow fractionation

ASP $=$ Alkaline Surfactant Polymer

$\mathrm{C}=$ concentration (ppm)

$\mathrm{C}^{*}=$ critical overlap concentration $(\mathrm{ppm})$

$\mathrm{D}=$ core dimeter $(\mathrm{cm})$

Deg $=$ mechanical degradation $(\%)$

$\mathrm{EOR}=$ enhanced oil recovery

GPC = gel permeation chromatography

HPAM = partially hydrolyzed polyacrylamide

$\mathrm{K}=$ flow consistency index $\left(\mathrm{cP.s}^{(\mathrm{n}-1)}\right)$

$\mathrm{K}_{\mathrm{abs}}=$ absolute permeability (Darcy)

$\mathrm{K}_{\mathrm{wf}}=$ absolute permeability after polymer flow (Darcy)

$\mathrm{K}_{\mathrm{wi}}=$ absolute permeability before polymer flow (Darcy)

$\mathrm{L}=$ core length $(\mathrm{cm})$

LSP = Low Salinity Polymer

$\mathrm{Mw}=$ molecular weight $(\mathrm{MDa})$

$\mathrm{MWD}=$ molecular weight distribution, dimensionless

$\mathrm{n}=$ flow behavior index, dimensionless

$\mathrm{PV}=$ pore volume, dimensionless

$\mathrm{Q}=$ injection flow rate $(\mathrm{cc} / \mathrm{min})$

$\mathrm{R}^{2}=$ coefficient of determination, dimensionless

$\mathrm{RF}=$ resistance factor, dimensionless

$\mathrm{RRF}=$ residual resistance factor, dimensionless

SAOS $=$ small-amplitude oscillatory shear

SEC = size exclusion chromatography

TDS $=$ total dissolved solids

$\mathrm{v}=$ interstitial velocity $(\mathrm{m} /$ day $)$

$\mathrm{v}_{\mathrm{c}}=$ onset of shear thickening $(\mathrm{m} /$ day $)$

$\mathrm{v}_{\mathrm{D}}=$ Darcy or Superficial velocity ( $\mathrm{m} /$ day)

$\Delta \mathrm{P}_{\mathrm{p}}=$ pressure drop during polymer flow (bar)

$\Delta \mathrm{P}_{\mathrm{w}}=$ pressure drop during water flow (bar)

$\eta_{\mathrm{e}}=$ effluent viscosity $(\mathrm{cP})$

$\eta_{\mathrm{i}}=$ injected solution viscosity $(\mathrm{cP})$

$\eta_{\mathrm{w}}=$ brine viscosity $(\mathrm{cP})$

$\phi=$ porosity, dimensionless

$\dot{\gamma}=$ shear rate $\left(\mathrm{s}^{-1}\right)$

\section{References}

1. Sorbie, K.S. Polymer-Improved Oil Recovery; Blackie and Son Ltd.: Glasgow, UK, 1991. [CrossRef]

2. Al Baqlani, S.; Singh, S.K.; Glasbergen, G.; van Elk, H. Polymer distribution concepts for large scale polymer floods in the sultanate of oman: Simplicity or flexibility? In Proceedings of the SPE EOR Conference at Oil and Gas West Asia, Muscat, Oman, 26-28 March 2018. [CrossRef]

3. Wilton, R.R.; Torabi, F. Rheological assessment of the elasticity of polymers for enhanced heavy oil recovery. In Proceedings of the SPE Heavy Oil Conference-Canada, Calgary, AB, Canada, 11-13 June 2013. [CrossRef]

4. Szabo, M.T. Molecular and microscopic interpretation of the flow of hydrolyzed polyacrylamide solution through porous media. In Proceedings of the Fall Meeting of the Society of Petroleum Engineers of AIME, San Antonio, TX, USA, 8-11 October 1972. [CrossRef]

5. Gao, C. Empirical correlations for viscosity of partially hydrolyzed polyacrylamide. J. Pet. Explor. Prod. Technol. 2013, 4, 209-213. [CrossRef] 
6. Hashmet, M.R.; Onur, M.; Tan, I.M. Empirical correlations for viscosity of polyacrylamide solutions with the effects of concentration, molecular weight and degree of hydrolysis of polymer. J. Appl. Sci. 2014, 14, 1000-1007. [CrossRef]

7. Gao, C. Viscosity of partially hydrolyzed polyacrylamide under shearing and heat. J. Pet. Explor. Prod. Technol. 2013, 3, 203-206. [CrossRef]

8. De Morais, S.C.; Cardoso, O.R.; de Carvalho Balaban, R. Thermal stability of water-soluble polymers in solution. J. Mol. Liq. 2018, 265, 818-823. [CrossRef]

9. Lewandowska, K. Comparative studies of rheological properties of polyacrylamide and partially hydrolyzed polyacrylamide solutions. J. Appl. Polym. Sci. 2007, 103, 2235-2241. [CrossRef]

10. Choi, S.K.; Sharma, M.M.; Bryant, S.; Huh, C. Ph-sensitive polymers for novel conformance-control and polymer-flood applications. SPE Reserv. Eval. Eng. 2010, 13, 926-939. [CrossRef]

11. Skauge, T.; Skauge, A.; Salmo, I.C.; Ormehaug, P.A.; Al-Azri, N.; Wassing, L.M.; Glasbergen, G.; Van Wunnik, J.N.; Masalmeh, S.K. Radial and linear polymer flow-Influence on injectivity. In Proceedings of the SPE Improved Oil Recovery Conference, Tulsa, OK, USA, 11-13 April 2016. [CrossRef]

12. Maerker, J.M. Shear degradation of partially hydrolyzed polyacrylamide solutions. Soc. Pet. Eng. J. 1975, 15, 311-322. [CrossRef]

13. Chang, H.L. Polymer flooding technology yesterday, today, and tomorrow. J. Pet. Technol. 1978, 30, 1113-1128. [CrossRef]

14. Needham, R.B.; Doe, P.H. Polymer flooding review. J. Pet. Technol. 1987, 39, 1503-1507. [CrossRef]

15. Standnes, D.C.; Skjevrak, I. Literature review of implemented polymer field projects. J. Pet. Sci. Eng. 2014, 122, 761-775. [CrossRef]

16. Sheng, J.J.; Leonhardt, B.; Azri, N. Status of polymer-flooding technology. J. Can. Pet. Technol. 2015, 54, 116-126. [CrossRef]

17. Skauge, A.; Shiran, B.S. Low salinity polymer flooding. In Proceedings of the IOR 2013-17th European Symposium on Improved Oil Recovery, St. Petersburg, Russia, 16-18 April 2013. [CrossRef]

18. Unsal, E.; Ten Berge, A.B.G.M.; Wever, D.A.Z. Low salinity polymer flooding: Lower polymer retention and improved injectivity. J. Pet. Sci. Eng. 2017, 163, 671-682. [CrossRef]

19. Zhu, Y.; Hou, Q.; Jian, G.; Ma, D.; Wang, Z. Current development and application of chemical combination flooding technique. Pet. Explor. Dev. 2013, 40, 96-103. [CrossRef]

20. Sheng, J.J. A comprehensive review of alkaline-surfactant-polymer (asp) flooding. Asian-Pac. J. Chem. Eng. 2014, 9, 471-489. [CrossRef]

21. Huh, C.; Pope, G.A. Residual oil saturation from polymer floods: Laboratory measurements and theoretical interpretation. In Proceedings of the SPE Symposium on Improved Oil Recovery, Tulsa, OK, USA, 20-23 April 2008. [CrossRef]

22. Xie, C.; Lv, W.; Wang, M. Shear-thinning or shear-thickening fluid for better eor?-A direct pore-scale study. J. Pet. Sci. Eng. 2017, 161, 683-691. [CrossRef]

23. Erincik, M.Z.; Qi, P.; Balhoff, M.T.; Pope, G.A. New method to reduce residual oil saturation by polymer flooding. SPE J. 2018, 23. [CrossRef]

24. Fan, J.C.; Wang, F.C.; Chen, J.; Zhu, Y.B.; Lu, D.T.; Liu, H.; Wu, H.A. Molecular mechanism of viscoelastic polymer enhanced oil recovery in nanopores. R. Soc. Open Sci. 2018, 5, 180076. [CrossRef]

25. Vik, B.; Kedir, A.; Kippe, V.; Sandengen, K.; Skauge, T.; Solbakken, J.; Zhu, D. Viscous oil recovery by polymer injection; impact of in-situ polymer rheology on water front stabilization. In Proceedings of the SPE Europec Featured at 80th EAGE Conference and Exhibition, Copenhagen, Denmark, 11-14 June 2018. [CrossRef]

26. Sorbie, K.S.; Roberts, L.J. A model for calculating polymer injectivity including the effects of shear degradation. In Proceedings of the SPE Enhanced Oil Recovery Symposium, Tulsa, OK, USA, 15-18 April 1984. [CrossRef]

27. Milton, H.W.; Argabright, P.A.; Gogarty, W.B. Eor prospect evaluation using field manufactured polymer. In Proceedings of the SPE California Regional Meeting, Ventura, CA, USA, 23-25 March 1983. [CrossRef]

28. Sieberer, M.; Clemens, T.; Peisker, J.; Ofori, S. Polymer flood field implementation-Pattern configuration and horizontal versus vertical wells. In Proceedings of the SPE Improved Oil Recovery Conference, Tulsa, OK, USA, 14-18 April 2018. [CrossRef]

29. Chauveteau, G.; Kohler, N. Influence of microgels in polysaccharide solutions on their flow behavior through porous media. Soc. Pet. Eng. J. 1984, 24, 361-368. [CrossRef] 
30. Thomas, A.; Gaillard, N.; Favero, C. Some key features to consider when studying acrylamide-based polymers for chemical enhanced oil recovery. Oil Gas Sci. Technol. 2013, 67, 887-902. [CrossRef]

31. Teeuw, D.; Hesselink, F.T. Power-law flow and hydrodynamic behaviour of biopolymer solutions in porous media. In Proceedings of the SPE Oilfield and Geothermal Chemistry Symposium, Stanford, CA, USA, 28-30 May 1980. [CrossRef]

32. Chauveteau, G.; Zaitoun, A. Basic rheological behavior of xanthan polysaccharide solutions in porous media: Effects of pore size and polymer concentration. In Enhanced Oil Recovery; Fayers, F.J., Ed.; Elsevier: Amstrdam, The Netherlands, 1981.

33. Morris, E.R.; Cutler, A.N.; Ross-Murphy, S.B.; Rees, D.A.; Price, J. Concentration and shear rate dependence of viscosity in random coil polysaccharide solutions. Carbohydr. Polym. 1981, 1, 5-21. [CrossRef]

34. Cannella, W.J.; Huh, C.; Seright, R.S. Prediction of xanthan rheology in porous media. In Proceedings of the SPE Annual Technical Conference and Exhibition, Houston, TX, USA, 2-5 October 1988. [CrossRef]

35. González, J.M.; Müller, A.J.; Torres, M.F.; Sáez, A.E. The role of shear and elongation in the flow of solutions of semi-flexible polymers through porous media. Rheol. Acta 2005, 44, 396-405. [CrossRef]

36. Noïk, C.; Delaplace, P.; Muller, G. Physico-chemical characteristics of polyacrylamide solutions after mechanical degradation through a porous medium. In Proceedings of the SPE International Symposium on Oilfield Chemistry, San Antonio, TX, USA, 14-17 February 1995. [CrossRef]

37. Southwick, J.G.; Manke, C.W. Molecular degradation, injectivity, and elastic properties of polymer solutions. SPE Reserv. Eng. 1988, 3, 193-201. [CrossRef]

38. Stavland, A.; Jonsbraten, H.; Lohne, A.; Moen, A.; Giske, N.H. Polymer flooding-Flow properties in porous media versus rheological parameters. In Proceedings of the SPE EUROPEC/EAGE Annual Conference and Exhibition, Barcelona, Spain, 14-17 June 2010. [CrossRef]

39. Wevera, D.A.Z.; Picchionia, F.; Broekhuisa, A.A. Polymers for enhanced oil recovery: A paradigm for structure-property relationship in aqueous solution. Prog. Polym. Sci. 2011, 36, 1558-1628. [CrossRef]

40. Zamani, N.; Kaufmann, R.; Kosinski, P.; Skauge, A. Mechanisms of non-newtonian polymer flow through porous media using navier-stokes approach. J. Dispers. Sci. Technol. 2015, 36, 310-325. [CrossRef]

41. Knight, J. Mechanical Shear Degradation of Polymers in Solution: A Review; Royal Aircraft Establishment: London, UK, 1976.

42. Caulfield, M.J.; Qiao, G.G.; Solomon, D.H. Some aspects of the properties and degradation of polyacrylamides. Chem. Rev. 2002, 102, 3067-3084. [CrossRef] [PubMed]

43. Hashmet, M.R.; Onur, M.; Tan, I.M. Empirical correlations for viscosity of polyacrylamide solutions with the effects of salinity and hardness. J. Dispers. Sci. Technol. 2014, 35, 510-517. [CrossRef]

44. Ryles, R.G. Chemical stability limits of water-soluble polymers used in oil recovery processes. SPE Reserv. Eng. 1988, 3, 23-34. [CrossRef]

45. Skauge, T.; Kvilhaug, O.A.; Skauge, A. Influence of polymer structural conformation and phase behavoir on in-situ viscosity. In Proceedings of the IOR 2015-18th European Symposium on Improved Oil Recovery, Dresden, Germany, 14-16 April 2015. [CrossRef]

46. Skauge, A.; Zamani, N.; Jacobsen, J.G.; Shiran, B.S.; Al-Shakry, B.; Skauge, T. Polymer flow in porous media: Relevance to enhanced oil recovery. Colloids Interfaces 2018, 2, 27. [CrossRef]

47. Hill, H.J.; Brew, J.R.; Claridge, E.L.; Hite, J.R.; Pope, G.A. The behavior of polymers in porous media. In Proceedings of the SPE Improved Oil Recovery Symposium, Tulsa, OK, USA, 22-24 April 1974. [CrossRef]

48. Hirasaki, G.J.; Pope, G.A. Analysis of factors influencing mobility and adsorption in the flow of polymer solution through porous media. Soc. Pet. Eng. J. 1974, 14, 337-346. [CrossRef]

49. Seright, R.S. The effects of mechanical degradation and viscoelastic behavoir on injectivity of polyacrylamide solutions. Soc. Pet. Eng. J. 1983, 23, 475-485. [CrossRef]

50. Heemskerk, J.; Rosmalen, R.; Janssen-van, R.; Holtslag, R.J.; Teeuw, D. Quantification of viscoelastic effects of polyacrylamide solutions. In Proceedings of the SPE Enhanced Oil Recovery Symposium, Tulsa, OK, USA, 15-18 April 1984. [CrossRef]

51. Muller, A.J.; Patruyo, L.G.; Montano, W.; Roversi-M, D.; Moreno, R.; Ramírez, N.E.; Sáez, A.E. Mechanical degradation of polymers in flows through porous media: Effect of flow path length and particle size. Appl. Mech. Rev. 1997, 50, S149-S155. [CrossRef]

52. Chauveteau, G.; Moan, M. The onset of dilatant behavior in non-inertial flow of dilute polymer-solutions through channels with varying cross-sections. J. Phys. Lett. 1981, 42, L201-L204. [CrossRef] 
53. Zamani, N.; Bondino, I.; Kaufmann, R.; Skauge, A. Effect of porous media properties on the onset of polymer extensional viscosity. J. Pet. Sci. Eng. 2015, 133, 483-495. [CrossRef]

54. Rock, A.; Hincapie, R.E.; Wegner, J.; Födisch, H.; Ganzer, L. Pore-scale visualization of oil recovery by viscoelastic flow instabilities during polymer eor. In Proceedings of the 19th European Symposium on Improved Oil Recovery, Stavanger, Norway, 24-27 April 2017. [CrossRef]

55. Kawale, D.; Marques, E.; Zitha, P.L.J.; Kreutzer, M.T.; Rossena, W.R.; Boukany, P.E. Elastic instabilities during the flow of hydrolyzed polyacrylamide solution in porous media: Effect of pore-shape and salt. Soft Matter 2017, 13, 765-775. [CrossRef]

56. Hu, Y.; Wang, S.Q.; Jamieson, A.M. Rheological and rheooptical studies of shear-thickening polyacrylamide solutions. Macromolecules 1995, 28, 1847-1853. [CrossRef]

57. Lake, L.W. Enhanced Oil Recovery; Prentice Hall, Inc.: Upper Saddle River, NJ, USA, 1989.

58. Seright, R.S.; Maerker, J.M.; Holzwarth, G. Mechanical degradation of polyacrylamides induced by flow through porous-media. ACS Polym. Prepr. 1981, 22, 30-33.

59. Puls, C.; Clemens, T.; Sledz, C.; Kadnar, R.; Gumpenberger, T. Mechanical degradation of polymers during injection, reservoir propagation and production-Field test results 8 th reservoir, austria. In Proceedings of the SPE Europec Featured at 78th EAGE, Vienna, Austria, 30 May-2 June 2016. [CrossRef]

60. Hunt, J.A.; Young, T.S.; Green, D.W.; Willhite, G.P. Size-exclusion chromatography in the measurement of concentration and molecular weight of some eor polymers. SPE Reserv. Eng. 1988, 3, 835-841. [CrossRef]

61. Dalsania, Y.; Doda, A.; Trivedi, J. Characterization of ultrahigh-molecular-weight oilfield polyacrylamides under different ph environments by use of asymmetrical-flow field-flow fractionation and multiangle-light-scattering detector. SPE J. 2018, 23, 48-65. [CrossRef]

62. Rodriguez, L.; Antignard, S.; Giovannetti, B.; Dupuis, G.; Gaillard, N.; Jouenne, S.; Bourdarot, G.; Morel, D.; Zaitoun, A.; Grassl, B. A new thermally stable synthetic polymer for harsh conditions of middle east reservoirs: Part ii. Nmr and size exclusion chromatography to assess chemical and structural changes during thermal stability tests. In Proceedings of the SPE Improved Oil Recovery Conference, Tulsa, OK, USA, 14-18 April 2018. [CrossRef]

63. Foshee, W.C.; Jennings, R.R.; West, T.J. Preperation and testing of partially hydrolyzed polyacrlamide solutions. In Proceedings of the SPE Annual Fall Technical Conference and Exhibition, New Orleans, LA, USA, 3-6 October 1976. [CrossRef]

64. Warner, H.R. Analysis of Mechanical Degradation Data on Partially Hydrolyzed Polyacrylamide Solutions; Society of Petroleum Engineers: Richardson, TX, USA, 1976.

65. Chauveteau, G. Molecular interperation of several different properties of flow of coiled polymer solutions through porous media in oil recovery conditions. In Proceedings of the SPE Annual Technical Conference and Exhibition, San Antonio, TX, USA, 4-7 October 1981. [CrossRef]

66. Jouenne, S.; Chakibi, H.; Levitt, D. Polymer stability after successive mechanical-degradation events. SPE J. 2018, 23, 18-33. [CrossRef]

67. Al-Shakry, B.; Skauge, T.; Shiran, B.S.; Skauge, A. Impact of mechanical degradation on polymer injectivity in porous media. Polymers 2018, 10, 742. [CrossRef]

68. Åsen, S.M.; Stavland, A.; Strand, D.; Hiorth, A. An experimental investigation of polymer mechanical degradation at $\mathrm{cm}$ and $\mathrm{m}$ scale. In Proceedings of the SPE Improved Oil Recovery Conference, Tulsa, OK, USA, 14-18 April 2018. [CrossRef]

69. Al Hashmi, A.R.; Al Maamari, R.S.; Al Shabib, I.S.; Masnsoor, A.M.; Zaitoun, A.; Al Sharji, H.H. Rheology and mechanical degradation of high-molecular-weight partially hydrolzed polyacrylamide during flow through capillaries. J. Pet. Sci. Eng. 2013, 105, 100-106. [CrossRef]

70. Dupas, A.; Henaut, I.; Rousseau, D.; Poulian, P.; Tabary, R.; Argillier, J.-F.; Aubry, T. Impact of polymer mechanical degradation on shear and extensional viscosities: Toward better injectivity forecasts in polymer flooding operations. In Proceedings of the SPE International Symposium on Oilfield Chemistry, The Woodlands, TX, USA, 8-10 April 2013. [CrossRef]

71. Zaitoun, A.; Makakou, P.; Blin, N.; Al-Maamari, R.S.; Al-Hashmi, A.R.; Abdel-Goad, M.; Al-Sharji, H.H. Shear stability of eor polymers. SPE J. 2012, 17, 335-339. [CrossRef]

72. Chauveteau, G.; Kohler, N. Polymer flooding: The essential elements for laboratory evaluation. In Proceedings of the SPE Improved Oil Recovery Symposium, Tulsa, OK, USA, 22-24 April 1974. [CrossRef] 
73. GRAESSLEY, W.W. The Entanglement Concept in Polymer Rheology; Springer: Berlin/Heidelberg, Germany, 1974.

74. Kulicke, W.M.; Haas, R. Flow behavior of dilute polyacrylamide solutions through porous media. 1. Influence of chain length, concentration, and thermodynamic quality of the solvent. Ind. Eng. Chem. Fundam. 1984, 23, 308-315. [CrossRef]

75. Mansour, A.M.; Al-Maarnari, R.S.; Al-Hashmi, A.S.; Zaitoun, A.; Al-Sharji, H. In-situ rheology and mechanical degradation of eor polyacrylamide solutions under moderate shear rates. J. Pet. Sci. Eng. 2014, 115, 57-65. [CrossRef]

76. Seright, R.S.; Seheult, M.; Talashek, T. Injectivity characteristics of eor polymers. SPE Reserv. Eval. Eng. 2009, 12, 783-792. [CrossRef]

77. Flew, S.; Sellin, R.H.J. Non-newtonian flow in porous media-a laboratory study of polyacrylamide solutions. J. Non-Newtonian Fluid Mech. 1993, 47, 169-210. [CrossRef]

78. Jouenne, S.; Heurteux, G. Flow of polymer solutions through porous media-prediction of mobility reduction from ex-situ measurements of elasticity. In Proceedings of the IOR 2017-19th European Symposium on Improved Oil Recovery, Stavanger, Norway, 24-27 April 2017. [CrossRef]

79. Gramain, P.; Myard, P. Elongational deformation by shear flow of flexible polymers adsorbed in porous media. Macromolecules 1980, 14, 180-184. [CrossRef]

80. Pelletier, E.; Viebke, C.; Meadows, J.; Williams, P.A. Dilute polyacrylamide solutions under uniaxial extensional flow. Langmuir 2003, 19, 559-565. [CrossRef]

81. Grigorescu, G.; Kulicke, W.-M. Prediction of viscoelastic properteis and shear stability of polymers in solution. In Advances in Polymer Science; Springer-Verlag: Berlin/Heidelberg, Germany, 2000.

82. Martin, F.D. Mechanical degradation of polyacrylamide solutions in core plugs from several carbonate reservoirs. SPE Form. Eval. 1986, 1, 139-150. [CrossRef]

83. Al-Hajri, S.; Mahmood, S.M.; Abdulelah, H.; Akbari, S. An overview on polymer retention in porous media. Energies 2018, 11, 2751. [CrossRef]

84. Seright, R.S. How much polymer should be injected during a polymer flood? SPE J. 2017, 22, 1-18. [CrossRef]

85. Han, X.-Q. Viscoelastic Behaviour of Polymer Molecules Retained in Porous Media; Society of Petroleum Engineers: Richardson, TX, USA, 1990.

86. Zhou, J.; Dong, Y.; Pater, C.J.; Zitha, P.L.J. Experimental study of the impact of shear dilation and fracture behavior during polymer injection for heavy oil recovery in unconsolidated reservoirs. In Proceedings of the Canadian Unconventional Resources \& International Petroleum Conference, Calgary, AB, Canada, 19-21 October 2010. [CrossRef]

87. Wever, D.A.Z.; Polgar, L.M.; Stuart, M.C.A.; Picchioni, F.; Broekhuis, A.A. Polymer molecular architecture as a tool for controlling the rheological properties of aqueous polyacrylamide solutions for enhanced oil recovery. Ind. Eng. Chem. Res. 2013, 52, 16993-17005. [CrossRef]

88. Wang, D.; Xia, H.; Liu, Z.; Yang, Q. Study of the mechanism of polymer solution with visco-elastic behavior increasing microscopic oil displacement efficiency and the forming of steady "oil thread" flow channels. In Proceedings of the SPE Asia Pacific Oil and Gas Conference and Exhibition, Jakarta, Indonesia, 17-19 April 2001. [CrossRef]

(C) 2018 by the authors. Licensee MDPI, Basel, Switzerland. This article is an open access article distributed under the terms and conditions of the Creative Commons Attribution (CC BY) license (http://creativecommons.org/licenses/by/4.0/). 